\title{
Modeling Hazard Rates as Functional Data for the Analysis of Cohort Lifetables and Mortality Forecasting
}

\author{
October 2008 \\ Jeng-Min Chiou \\ Institute of Statistical Science, Academia Sinica \\ 128 Sec. 2 Academia Rd., Taipei 11529, Taiwan. E-mail: jmchiou@stat.sinica.edu.tw \\ Hans-Georg Müller \\ Department of Statistics, University of California \\ One Shield Ave., Davis, CA 95616, USA. E-mail: mueller@wald.ucdavis.edu
}

Author's Footnote:

Jeng-Min Chiou is Associate Research Fellow, Institute of Statistical Science, Academia Sinica, Taipei 11529, Taiwan (E-mail: jmchiou@stat.sinica.edu.tw). Hans-Georg Müller is Professor, Department of Statistics, University of California, Davis, CA 95616 (E-mail:

mueller@wald.ucdavis.edu). We are extremely grateful to the Associate Editor for patience, constructive feedback and detailed recommendations, and also wish to thank six anonymous referees for careful reading and helpful suggestions that substantially improved the paper. This research was supported in part by grants from National Science Council (NSC95-2118M001013MY3), and National Science Foundation (DMS-0354448, DMS-0505537 and DMS-086199). 


\title{
Modeling Hazard Rates as Functional Data for the Analysis of Cohort Lifetables and Mortality Forecasting
}

\begin{abstract}
As world populations age, the analysis of demographic mortality data and demographic predictions of future mortality have met with increasing interest. The study of mortality patterns and the forecasting of future mortality with its associated impacts on social welfare, health care and societal planning has become a more pressing issue. An ideal set of data to study patterns of change in long-term mortality is the well-known historical Swedish cohort mortality data, due to its high quality and long span of more than two centuries. We explore the use of functional data analysis to model these data and to derive mortality forecasts. Specifically, we address the challenge of flexibly modeling these data while including the effect of the birth year by regarding log-hazard functions, derived from observed cohort lifetables, as random functions. A functional model for the analysis of these cohort log hazard functions, extending functional principal component approaches by introducing time-varying eigenfunctions, is found to adequately address these challenges. The associated analysis of the dependency structure of the cohort log hazard functions leads to the concept of time-varying principal components of mortality. We then extend this analysis to mortality forecasting, by combining prediction of incompletely observed log-hazard functions with functional local extrapolation, and demonstrate these functional approaches for the Swedish cohort mortality data.
\end{abstract}

KEY WORDS: Eigenfunction; Force of mortality; Functional data analysis; Log hazard function; Prediction; Principal component; Swedish mortality; Time-varying modeling. 


\section{INTRODUCTION}

Assessing demographic trends in mortality is of growing interest due to the demographic impacts of aging and extended longevity on the future viability of social security and retirement systems and the associated societal changes. Such trends not only raise basic biodemographic questions about the nature and the limits of human lifespan (Oeppen and Vaupel, 2002; Vaupel et al., 1998) but also about the demographic future of aging societies. Two issues are of interest: the analysis of recurring mortality patterns and their structure, and the prediction and forecasting of future cohort mortality.

The starting point of our mortality analysis are Swedish female mortality data for year-of-birth cohorts, available from the Human Mortality Database (www.mortality.org), see Glei, Lundström and Wilmoth (2006). Thanks to the exceptional quality of official statistics and record keeping, the Swedish cohort mortality data are thought to be among the most reliable long-term longitudinal cohort mortalities available. To date, this data collection provides information on completely recorded mortalities for longitudinally observed birth cohorts born in the years 1751 to 1975 , spanning more than 200 years. For the domain of the log hazard trajectories we choose ages 0 to 80 for our analysis, so as to include as many completely observed log hazard functions as possible.

Figure 1 displays estimated log-hazard functions for eight cohorts, over a wide range of birth years. It is obvious that dramatic changes in mortality have occurred over historic periods, a well-known phenomenon (Oeppen and Vaupel 2002, Vaupel and Canudas Romo 2003). The striking patterns of lifetable evolution with the overall lowering of mortality from the past to the present and the fairly substantial variation in the shape of the loghazard functions pose two challenges: To model these data by viewing them as a sample of log-hazard functions, and to include the birth year of the cohorts as an important covariate in the modeling, as a successful model needs to flexibly adapt to the shape changes that occur in the Swedish lifetables over the centuries. Due to its inherently nonparametric 
nature, a functional data approach is expected to address these challenges.

To understand and model the complex shapes and shape changes of the cohort log hazard functions for the Swedish mortality data, an assessment of the dependency structure of mortality, e.g., through the covariance of mortality between different ages within the same cohort, is of prime interest. Thus we are interested in a model for these data that combines the influence of birth year with a model of intra-cohort mortality dependence, allowing for a dependence structure that along with mean functions may change over time. We refer to this concept as "time-varying principal components of mortality". In addition to modeling the Swedish cohort lifetables, we are also interested in taking advantage of the specific features of this approach (a) to predict mortality for those cohorts that are incompletely observed; and (b) to forecast mortality for cohorts born in the future. Matlab code that implements the proposed procedures is available in an online Supplement to this article.

In this article we pursue two main goals: To demonstrate a novel and useful analysis of the mortality structure inherent in the completely observed cohorts of the Swedish cohort lifetables; and to harness these approaches to predict and forecast cohort mortality. In Section 2 we present the "moving windows approach", devised to meet the first challenge, while details on implementation of the moving-windows modeling approach and the data analysis are provided in Section 3. What we can learn from these data for predicting cohort $\log$ hazard functions from incompletely observed cohort mortalities and for forecasting future mortality is the topic of Section 4, followed by concluding remarks (Section 5). 


\section{MODELING COHORT LOG HAZARD FUNCTIONS FOR SWEDISH COHORT LIFETABLES AS FUNCTIONAL DATA}

\subsection{Lifetable Data and Hazard Functions}

Several classical parametric models are available for modeling cohort log-hazard functions on their entire domain or on suitable subdomains, usually implemented by maximum likelihood. These include the Heligman-Pollard empirical method (Heligman and Pollard, 1980), the Gompertz model, and variants. We were unable to obtain good fits for the Swedish mortality data with the relatively flexible Heligman-Pollard model. The large heterogeneity of the hazard functions over the centuries (Figure 1) makes parametric modeling with its inherent shape restrictions a difficult and unwieldy proposition. Thus faced with the need for an alternative more flexible nonparametric approach for these data, we first obtain a log hazard function nonparametrically from the lifetable data of each cohort.

Demographic lifetable data can be represented by the pairs $\left(N_{j-1}, d_{j}\right)$, where $N_{j-1}$ is the number of subjects alive (i.e., at risk) at the beginning of the $j$ th lifetable interval (defining $N_{0}$ as the total number of subjects at risk in the beginning of the first lifetable interval) and $d_{j}$ is the number of deaths occurring during the interval. These data are collected for $j=1,2, \ldots$ until $N_{j-1}=0$ occurs, i.e., until all subjects in a cohort have died. The corresponding $j$ th time interval of the lifetable is assumed to be $[(j-1) \Delta, j \Delta]$. The interval length $\Delta$ may vary. In the Swedish mortality lifetables it is one year. If $t_{j}$ denotes the midpoint of the $j$ th interval so that the $j$ th lifetable interval is $\left[t_{j}-\Delta / 2, t_{j}+\Delta / 2\right]$, the death rate $q$ is $q\left(t_{j}\right)=d_{j} / N_{j-1}$, and the central death rate $\tilde{q}_{c}$ (also denoted as $m$ in some demographic and actuarial contexts, e.g., Elandt-Johnson and Johnson, 1980) is

$$
\tilde{q}_{c}\left(t_{j}\right)=d_{j} /\left\{\left(N_{j-1}+N_{j}\right) \Delta / 2\right\}
$$


When one targets the hazard function (force of mortality), defined as

$$
h(t)=\lim _{\Delta \rightarrow 0} \frac{1}{\Delta} \operatorname{Pr}(T \in[t, t+\Delta] \mid T>t)
$$

where $T$ is a continuous random variable denoting age-at-death (survival time) for a subject, discretization biases arise due to the aggregated nature of the lifetable data. Wang, Müller and Capra (1998) and Müller, Wang and Capra (1997) proposed a transformation approach aimed at minimizing this discretization bias, which we adopt here. The goal is

to find a transformation $\psi$ of the central death rate $\tilde{q}_{c}$ such that $\psi\left(\tilde{q}_{c}(t)\right)=\int_{t-\Delta / 2}^{t+\Delta / 2} h(t) d t$ which provides the closest possible approximation to $h(t)$, using aggregated information about $h$ in the interval $[t-\Delta / 2, t+\Delta / 2]$, as $\Delta \rightarrow 0$. This transformation turns out to be

$$
\psi(x)=\frac{1}{\Delta} \log \frac{2+x \Delta}{2-x \Delta} .
$$

Applying this transformation to the central death rate then leads to

$$
Y\left(t_{j}\right)=\log \left\{\psi\left(\tilde{q}_{c}\left(t_{j}\right)\right)\right\}=Z\left(t_{j}\right)+\epsilon_{j}
$$

as "observations at $t_{j}$ " of the underlying $\log$-hazard function $Z(t)=\log \{h(t)\}$, contaminated with zero mean observation errors, $\epsilon_{j}$, that reflect deviations of the observed values from the underlying hazard trajectories due to the discretization and random nature of the counts in each lifetable interval of length $\Delta$.

\subsection{Functional Principal Components for Cohort Log Hazard Functions}

The seminal work of Lee and Carter (1992) for modeling and forecasting long-term trends in mortality rates demonstrates the importance of statistical approaches (see also Dellaportas, Smith and Stavropoulos, 2001; Renshaw and Haberman 2003; Currie, Durban and Eilers, 2004; Li, Lee and Tuljapurkar, 2004; Wong-Fupuy and Haberman, 2004; Li and Lee, 2005; Booth, 2006; Debón, Montes and Sala, 2006). To overcome the problems and inherent limitations encountered with parametric modeling, we develop here a functional 
data analysis (FDA) approach, where the functional data are the log hazard functions. This approach is nonparametric and imposes only minimal assumptions on the data.

Ramsay and Silverman $(2002,2005)$ provide an excellent overview of the methodological foundations of FDA, with shorter reviews in Rice (2004) and Müller (2005). Our approaches are motivated by response function models, with functional responses and vector predictors (Chiou, Müller and Wang, 2004). Other functional approaches relevant for demographic data include Park, Choi and Kim (2006), who proposed to model with two random processes, and Hyndman and Ullah (2007), who developed a functional approach for forecasting mortality and fertility rates, combining FDA and time series techniques with robustness features. A time-varying functional regression model for biodemographic and general event-history data for which a longitudinal subject-specific covariate is available was developed in Müller and Zhang (2005), and conditional functional principal component analysis closely related to our approach appeared recently in Cardot (2007). Previous work along these lines also includes the modeling of mortality functions derived from lifetable data as random functions in Capra and Müller (1997), the functional modeling of density families in Kneip and Utikal (2001), and a smooth random-effects model for random density functions (Chiou and Müller, 2001). Applications of FDA cover a vast range from ergonomics (Faraway, 1997) to the analysis of gene expression data (Zhao, Marron and Wells, 2004, Müller, Chiou and Leng, 2008).

Functional Principal Component Analysis (FPCA) provides a decomposition into a systematic part that corresponds to the overall mean function, and a random part; it is motivated by the Karhunen-Loève expansion (Ash and Gardner, 1975) for stochastic processes. Briefly, for the cohort-specific $\log$ hazard functions $Z(t)=\log \{h(t)\}, t \in[0, T]$, (see eq. (1),(2)), the Karhunen-Loève representation for these random log hazard functions 
is

$$
Z(t)=\mu(t)+\sum_{k=1}^{\infty} \xi_{k} \rho_{k}(t)
$$

where $\mu(t)$ is the overall mean function, and $\rho_{k}(t)$ is the $k$ th orthonormal eigenfunction of the auto-covariance operator, a linear operator in the space of square integrable functions, that is generated by the covariance kernel $G\left(t_{1}, t_{2}\right)=\operatorname{cov}\left(Z\left(t_{1}\right), Z\left(t_{2}\right)\right)$. The $\xi_{k}$ are the functional principal component scores, from now on referred to as principal components. These are random variables $\xi_{k}=\int_{0}^{T}\left(Z(t)-\mu_{0}(t)\right) \rho_{k}(t) d t$, with $E \xi_{k}=0$ and $\operatorname{var}\left(\xi_{k}\right)=\lambda_{k}$. Here $\lambda_{k}$ is the eigenvalue corresponding to the eigenfunction $\rho_{k}, k=1,2, \ldots$ (see Ash and Gardner, 1975). As a result, the covariance of the cohort log hazard functions at any two ages $t_{1}$ and $t_{2}$ can be expressed as

$$
G\left(t_{1}, t_{2}\right)=\sum_{k=1}^{\infty} \lambda_{k} \rho_{k}\left(t_{1}\right) \rho_{k}\left(t_{2}\right)
$$

It is easiest to understand FPCA as an extension of classical multivariate PCA, which is defined in a Euclidean vector space of random vectors of a fixed dimension $p \geq 1$, to the case where the random vectors are replaced by random functions; in our case this role is played by the cohort log hazard functions. The appropriate vector space is then the infinite-dimensional Hilbert space of square integrable functions $L^{2}$. In this extension, vectors become functions, matrices become linear operators in Hilbert space, and in a more formal approach one needs to define in which way the infinite sums that one considers in (3) and (4) converge to their limits. Both representations (3) and (4) have close analogues in the vector PCA case, where they are discussed in textbooks such as Johnson and Wichern (2002, Sections 8.2, 8.3). We note that the interpretation of the principal components $\xi_{k}$ is essentially the same in functional and vector cases.

The cohort log-hazard functions (2) for $n$ observed cohort lifetables $i=1, \ldots, n$ are then represented as

$$
Y_{i}\left(t_{j}\right)=Z_{i}\left(t_{j}\right)+\epsilon_{i j}=\mu\left(t_{j}\right)+\sum_{k=1}^{\infty} \xi_{k i} \rho_{k}\left(t_{j}\right)+\epsilon_{i j}, \quad 1 \leq i \leq n, 1 \leq j \leq m,
$$


where $m=[T / \Delta]$ and the random errors $\epsilon_{i j}$ are uncorrelated and independent of $\xi_{k i}$, with $E\left(\epsilon_{i j}\right)=0, \operatorname{var}\left(\epsilon_{i j}\right)=\sigma_{\epsilon}^{2}$. The covariances of the data (2) are

$$
\operatorname{cov}\left(Y\left(t_{j}\right), Y\left(t_{l}\right)\right)=\operatorname{cov}\left(Z_{i}\left(t_{j}\right), Z_{i}\left(t_{l}\right)\right)+\sigma_{\epsilon}^{2} \delta_{j l}
$$

where $\delta_{j l}=1$ if $j=l$ and 0 otherwise. The above representations form the basis for the proposed mortality modeling and forecasting. According to (5), the additional measurement error is reflected in a non-smooth ridge that sits atop the diagonal of the covariance surface; compare Staniswalis and Lee (1998).

\subsection{Moving Windows Approach}

A major challenge for modeling the Swedish cohort lifetables is how to appropriately include the clearly discernible influence of the birth year of the cohort. Preliminary analysis revealed that not only the mean of the cohort log hazard functions but also their internal dependence structure depend on birth year, which is not surprising as the birth years of the cohorts extend over more than two centuries. In light of (4), it is then plausible that the eigenfunctions in the functional principal component expansion of the cohort loghazard functions not only are smooth but also depend smoothly on the covariate birth year of the cohort.

To model the dependence of both mean and eigenfunctions on the birth year, we consider a moving windows approach. A key feature is the inclusion of both time-varying mean functions and time-varying eigenfunctions in the representation (3). The stochastic (covariance and mean) structure of the cohort log hazard functions is assumed to change smoothly and slowly as a function of cohort birth year $X$. This implies that this structure is approximately constant for those log-hazard functions for which $X$ falls into a window around a specified level $x_{0}$. Given such a window $\mathcal{W}\left(x_{0}\right)$ around $x_{0}$, we then aggregate these cohort log hazard functions and treat them as an i.i.d. sample of random functions. 
The mean and covariance structure of these random functions is determined by the window $\mathcal{W}\left(x_{0}\right)$.

Formally, assume $x_{0}$ is a given birth year and $\omega>0$ is a window width. Define the windows $\mathcal{W}\left(x_{0}\right)=\left\{x_{i}: x_{i} \in\left[\max \left(x_{1}, x_{0}-\omega\right), \min \left(x_{n}, x_{0}+\omega\right)\right]\right\}$, where we assume that the covariate levels (birth years) are discrete and ordered by size, i.e., $x_{1} \leq x_{2} \leq \ldots \leq x_{n}$. In our application, $x_{1}=1751, \ldots$, and $x_{n}=1914$ for complete lifetables. Motivated by (3), we consider a conditional version for the cohort $\log$ mortality functions $Z$, whose covariate falls into the window $\mathcal{W}\left(x_{0}\right)$, i.e.,

$$
Z_{x_{0}}(t)=\mu_{x_{0}}(t)+\sum_{k=1}^{\infty} \xi_{k, x_{0}} \rho_{k, x_{0}}(t)
$$

where $\mu_{x_{0}}$ and $\rho_{k, x_{0}}$ are mean and covariance functions as in (3), however restricted to the cohort $\log$ mortality functions $Z_{i}$ for which $x_{i} \in \mathcal{W}_{0}$, and

$$
\xi_{k, x_{0}}=\int_{0}^{T}\left(Z_{x_{0}}(t)-\mu_{x_{0}}(t)\right) \rho_{k, x_{0}}(t) d t, \quad E\left(\xi_{k, x_{0}}\right)=0, \quad E\left(\xi_{k, x_{0}}^{2}\right)=\lambda_{k, x_{0}} .
$$

For the covariance function $G_{x_{0}}(s, t)=\operatorname{cov}\left(Z_{x_{0}}(s), Z_{x_{0}}(t)\right)$ of $\log$ hazard functions $Z_{x_{0}}$, we then have $G_{x_{0}}(s, t)=\sum_{k=1}^{\infty} \lambda_{k, x_{0}} \rho_{k, x_{0}}(s) \rho_{k, x_{0}}(t)$.

Representation (6) provides a windowed version of FPCA that turns out to be quite appropriate for modeling the Swedish cohort log hazard functions. This approach entails the necessary flexibility to model these cohort lifetables and their changing patterns over the centuries, without suffering from the drawbacks of parametric approaches. It addresses the two major modeling challenges posed by the Swedish lifetable data: Flexibly modeling the sample of log-hazard functions corresponding to the cohort lifetables, and incorporating the dependency on the birth year of the cohort for both eigenfunctions and mean structure of the functions. 


\section{PRINCIPAL COMPONENTS OF MORTALITY FOR THE SWEDISH COHORT LIFETABLES}

\subsection{Implementing the Moving Windows Approach}

Model (6) is nonparametric, as no assumption is made on the shape of the hazard functions or the eigenfunctions. The mean function $\mu_{x_{0}}(t)$ is estimated for each single year of age from 0 to $80, t$ denoting age of subjects in each cohort, and varies in dependence on the birth year $x_{0}$ of the cohort. The dependence on birth year is implemented by averaging over the cohorts with birth year in the window around the birth year $x_{0}$ of a given cohort. The eigenfunctions $\rho_{k, x_{0}}(t)$ characterize the main directions of the deviations of the log hazards of individual cohorts from the mean log hazard function within the window. These random deviations are thus decomposed in these eigenfunctions and principal components $\xi_{k, x_{0}}$, which vary according to the size of these deviations for specific cohorts, and play the role of cohort-specific random effects. The principal components need to be estimated separately for each cohort with birth year within the given window. The interpretation of the eigenfunctions is analogous to that of eigenvectors in multivariate PCA. In our model, the eigenfunctions vary in dependence on the birth year of the cohort, as they are calculated only for the cohorts with birth year falling into the window around $x_{0}$. The essence of fitting this model is that one first obtains the mean log-hazard functions by averaging over cohorts with similar birth years, and then the residuals between the observed log-hazards for a specific cohort and this mean function are decomposed via a functional version of principal component analysis.

Accordingly, estimation of the model component functions $\mu_{x_{0}}, \rho_{k, x_{0}}$ and of corresponding cohort log hazard functions in model (6) is based on the data of cohorts with birth years $x_{i}$ falling into the window $\mathcal{W}\left(x_{0}\right)$. For estimation of the mean function $\mu_{x_{0}}$, we use the locally weighted least squares smoothing method applied to the pooled measurements made on all cohort log hazard functions of the cohorts within the window. The 
necessary smoothing parameter can be chosen by various methods, e.g., cross-validation (Rice and Silverman, 1991). Eigenfunctions $\rho_{k, x_{0}}$ are obtained by first estimating the auto-covariance function of $Z_{x_{0}}$, for which we adopt the techniques proposed in Yao et al. (2003), i.e., smoothing the empirical covariances with special handling for the diagonal which is removed in the initial smoothing steps as it may be contaminated by the measurement errors. From smooth estimates of the covariance function $\operatorname{cov}(Z(s), Z(t))$, one then numerically derives estimates $\hat{\lambda}_{k}$ and $\hat{\rho}_{k, x_{0}}$ of eigenvalues and eigenfunctions by discretizing and applying the corresponding procedures for matrices. Finally, fitted covariance surfaces are obtained by

$$
\hat{G}_{x_{0}}(s, t)=\sum_{k=1}^{L} 1_{\left\{\hat{\lambda}_{k, x_{0}}>0\right\}} \hat{\lambda}_{k, x_{0}} \hat{\rho}_{k, x_{0}}(s) \hat{\rho}_{k, x_{0}}(t) ;
$$

these are guaranteed to be symmetric and nonnegative definite. Fitted correlation surfaces are an immediate consequence. Here $L$ denotes the number of included components.

Once estimates for these fixed component functions have been found, the fitting of cohort log hazard functions requires one to predict the cohort-specific principal components $\xi_{k, x_{0}}, k \geq 1$ in model (6). Since lifetables correspond to the log hazard observations in eq. (2) on a regular dense grid, one may obtain estimates by approximating integrals in eq. (7) with sums,

$$
\tilde{\xi}_{k, x_{0}}=\Delta \sum_{j=1}^{m}\left(Y_{x_{0}}\left(t_{j}\right)-\hat{\mu}_{x_{0}}\left(t_{j}\right)\right) \hat{\rho}_{k, x_{0}}\left(t_{j}\right),
$$

where $\Delta$ is the length of the lifetable intervals with $\Delta=1$ year for the Swedish lifetables, and $Y_{x_{0}}\left(t_{j}\right)=Y\left(t_{j}\right)$, as in eq. (2), for the cohort born at time $x_{0}$. These estimates may be further refined by a shrinkage step as in Yao et al. (2003) to adjust for measurement errors, leading to

$$
\hat{\xi}_{k, x_{0}}=\frac{\hat{\lambda}_{k, x_{0}}}{\hat{\lambda}_{k, x_{0}}+\hat{\theta} / n_{x_{0}}} \tilde{\xi}_{k, x_{0}},
$$

where $n_{x_{0}}$ is the number of available observations for the cohort, $n_{x_{0}}=m=81$ for all 
$x_{0}$ for the Swedish lifetable data. The estimate $\hat{\theta}$ of the shrinkage parameter $\theta$ can be obtained by leave-one-curve-out cross-validation, as explained in Yao et al. (2003).

These steps result in fitted cohort log hazard functions

$$
\hat{Z}_{x_{0}}(t)=\hat{\mu}_{x_{0}}(t)+\sum_{k=1}^{L} \hat{\xi}_{k, x_{0}} \hat{\rho}_{k, x_{0}}(t),
$$

for some predetermined number $L$, reflecting the number of included components and random effects. Results on the consistency of estimated functions $\hat{Z}_{x_{0}}(11)$ are provided in Appendix A.1. The fitted cohort log hazard functions in (11) depend on two auxiliary parameters, namely, the size $\omega$ of the window $\mathcal{W}\left(x_{0}\right)=\mathcal{W}\left(x_{0} ; \omega\right)$, and the dimension L. For their selection, we adopt data-based choices described in Appendix A.2, which worked well for the Swedish cohort lifetables. These selectors combine a "fraction of variance explained" criterion for the choice of $L$ with the quality of the approximation of the local empirical covariances within the bins for the choice of $\omega$. Too small as well as too large bin sizes $\omega$ are associated with increased approximation errors. A plot of the size of the deviation of fitted versus raw covariances, as quantified in (A.2), is displayed in Figure 2, leading to the choices $\omega^{*}=11$ and $L^{*}=2$.

\subsection{Time-Varying Principal Components of Mortality}

Analyzing the Swedish lifetable data with the moving windows approach, the time-varying feature of the principal components of mortality for the Swedish log-hazard functions over the centuries is confirmed by Figure 3, comparing the internal dependence structure of the cohort log hazard functions for cohorts born in 1820 and in 1900. The upper panels of Figure 3 illustrate the smoothed covariance functions. The covariances for the 1820 cohorts are generally lower, while the overall structure is similar although clearly not the same. This becomes more obvious from the fitted correlation surfaces obtained as $\widehat{\operatorname{corr}}(s, t)=\hat{G}_{x_{0}}(s, t) /\left\{\hat{G}_{x_{0}}(s, s) \hat{G}_{x_{0}}(t, t)\right\}^{1 / 2}$ derived from (8) which are shown in the 
middle panels of Figure 3. We find nearly constant high correlation among all higher age mortalities, and lower correlations for young age mortalities with older age mortalities.

Interestingly, the range of older ages among which mortality correlations are higher is shorter for the 1820 cohort, where this range includes ages 20 and higher, than it is for the 1900 cohort, where it includes ages 15 and above. This may point to a more general phenomenon of increasing systematic influences and less randomness affecting log-hazard functions of more recently born individual cohorts, and especially so for the log-hazard functions at older ages. This interpretation is also supported by the eigenvalues and the fractions of variance explained for the two selected components (1820: 0.2794 (89.73\%), 0.0289 (9.28\%); 1900: 2.5796 (98.17\%), 0.0427 (1.63\%)), meaning that more of the observed variation in log-hazard functions is explained for the more recent cohorts, and especially more of the variation is explained by the first eigenfunction. This points to increasing stability of the more recent log-hazard functions.

The associated eigenfunctions for the 1820 and 1900 cohorts are shown in the bottom panels. They display overall similar shapes but shifted trough and peak locations, as well as changes in amplitude. A local peak is visible in the first eigenfunction, corresponding to increased overall variability of mortality just before age 60 in the 1820 cohort and just before age 40 in the 1900 cohort, so the area of maximum mortality variability has moved earlier for adult mortality. The variability of infant mortality has declined from 1820 to 1900. These features can also be discerned in the covariance surfaces in the top panels. The variability of mortality reflected in the second principal component and visualized in the second eigenfunctions has not changed much from 1820 to 1900. Overall, we find smooth time-variation of the eigenfunctions, supporting the concept of slowly evolving principal components of mortality. This lends support to the assumption that eigenfunctions are approximately constant within suitably chosen windows $\mathcal{W}\left(x_{0}\right)$, but not constant over the centuries. 
The effect of time-varying principal components of mortality on the evolution of the shapes of log-hazard functions over the 18th-19th centuries is illustrated further through the fitted cohort log hazard functions (11) in Figure 4 for 1751-1880 (left panel) and 18811910 (right panel). The curves at lower positions correspond to more recent calendar years, respectively, for both plots. The curves in the right panel (1881-1910) correspond to lower log-hazard functions as compared to those in the left panel (1751-1880). It is interesting that before year 1880 the log-hazard functions display a single trough, at around age 12, while there are dual troughs in the log-hazard functions after year 1880, with a second trough of mortality becoming apparent at age 40. These troughs become more pronounced for later birth years. This feature can be alternatively viewed as a peak of mortality developing around age 20, the "mortality hump" which, for example, motivated the Heligman-Pollard law. The biggest trend though is the universal decline in mortality across all ages. Interestingly, the sizes of the overall changes between 1751 to 1880, a 130 year period, and between 1881 and 1910, a 20 year period, are comparable, pointing towards a secular acceleration in the rate of change of mortality dynamics.

The moving windows approach naturally addresses the time-varying characteristics of the principal components of mortality that we find for these log-hazard functions. The relative performance when comparing moving windows with a global principal component approach, where one would choose one set of eigenfunctions for the entire sample (full window), irrespective of year of birth, can be quantified through the leave-one-curve-out prediction errors,

$$
\sum_{x_{i} \in \mathcal{I}_{Z}} \sum_{j=1}^{m} \Delta\left(Z_{x_{i}}(t)-\hat{Z}_{x_{i}}^{(-i)}\left(t_{j}\right)\right)^{2}, \quad \text { where } \quad \hat{Z}_{x_{i}}^{(-i)}(t)=\hat{\mu}_{x_{i}}^{(-i)}(t)+\sum_{k=1}^{L} \hat{\xi}_{k, x_{i}}^{(-i)} \hat{\rho}_{k, x_{i}}^{(-i)}(t)
$$

and the estimates $\hat{\rho}_{k, x_{i}}^{(-i)}(t)$ and $\hat{\mu}_{x_{i}}^{(-i)}(t)$ are obtained by leaving out the $i$ th mortality trajectory, while $\hat{\xi}_{k, x_{i}}^{(-i)}=\Delta \sum_{j=1}^{m}\left(Y_{x_{i}}\left(t_{j}\right)-\hat{\mu}_{x_{i}}^{(-i)}\left(t_{j}\right)\right) \hat{\rho}_{k, x_{j}}^{(-i)}\left(t_{j}\right)$, following (9), and we choose $\mathcal{I}_{Z}=[1760,1900]$. 
Table 1 presents the results for full window approaches with models including all available lifetables in one analysis and the local window approach that includes data within the moving window. Full window approaches include the model in (11) but with the window stretching over all data, and the functional regression model with smooth random effects (Chiou et al., 2003) where the functional principal component scores are smooth function of the covariate (the calendar year). The local window approach is based on model (13) with the optimal bin size chosen as 11. We found that increasing the number of components beyond 5 or 6 does not further reduce the prediction errors, which stay approximately constant. More importantly, the prediction errors using the moving windows approach are always smaller then the global approaches, irrespective of the number of components included in the models.

\section{PREDICTING AND FORECASTING LOG-HAZARD FUNCTIONS}

\subsection{Overview}

Forecasting future mortality is of major interest for demographic projections and societal planning, affecting social security and health care providers, among many others. Using FDA approaches, Hyndman and Ullah (2007) proposed a two-step robust principal component method of forecasting by predicting coefficients corresponding to the principal components, through time series models such as the traditional ARIMA models and exponential smoothing time series models (Hyndman et al., 2002). Building on the moving windows approach described above, we consider here a different, completely nonparametric approach. Notably, our approach does not rely on parametric time series structural assumptions for the forecasts. A pertinent problem is that of predicting cohort log hazard functions for cohorts for which complete log hazard functions have not been observed yet, since the birth of the cohort members is too recent so that cohort members are still alive.

We will address this prediction problem by a conditional expectation method intro- 
duced in Section 4.2. This approach combines the information available in all cohort log mortality functions, whether they have been completely or partially observed, for the purpose of obtaining both mean and covariance functions in windows $\mathcal{W}\left(x_{0}\right)$. Window-specific eigenvalues and eigenfunctions can be obtained subsequently. While the conditional approach is suitable for the prediction of incompletely observed cohort log hazard functions, our ultimate goal is to forecast log-hazard functions for cohorts that have not been born yet, or have been born very recently, and for which the cohort log hazard functions are essentially unobserved. We refer to this challenge as the forecasting problem and approach it with a functional local linear extrapolation method, applied to the predicted mean and eigenfunctions for the incompletely observed cohorts that were obtained in the preliminary prediction step. For the construction of associated prediction intervals we utilize the bootstrap.

Throughout this section we use the following notation: $x^{*}$ denotes the birth year of a cohort for which one intends to forecast or predict mortality. If the target year $x^{*}$ is beyond the birth year of the last at least partially observed cohort which we denote by $x_{n}^{*}$, i.e., if $x^{*}>x_{n}^{*}$, then this is a forecasting problem. We denote a target year $x^{*}$ that leads to a forecasting problem by $x_{F}^{*}$. If $x^{*} \leq x_{n}^{*}$, then $x^{*}$ is the birth year of an incompletely observed cohort, leading to a prediction problem, for which we denote the target year $x^{*}$ by $x_{P}^{*}$. From the Human Mortality Database as of 2006, all Swedish cohort mortalities for cohorts born until 1975 are available until 2004. This means $x_{n}^{*}=1975$, and the 1975 cohort has been observed for 29 years and thus is incompletely observed, as are all cohorts born after 1924, given the chosen domain of $[0,80]$. Then, for all cohorts born after 1975, we face a forecasting problem, while for cohorts born between 1925 and 1974 we have a prediction problem. The prediction problem is addressed in Section 4.2 and the forecasting problem in Section 4.3. 


\subsection{Predicting Incompletely Observed Cohort Log Hazard Functions}

The conditional method which we describe here borrows strength from all observed functions in order to overcome the problem of incomplete data for a given cohort. The idea is to combine and include all information available in the various log hazard functions which fall into the windows $\mathcal{W}\left(x_{0}\right)$, whether they have been completely or only partially observed, for the purpose of obtaining both mean and covariance function estimates, followed by the estimation of window-specific eigenvalues and eigenfunctions. The conditional regression method then leads to predictions of the principal components for cohorts with incompletely or partially observed log hazard functions. It is based on three assumptions: Gaussian measurement errors and Gaussian principal components, dense overall designs when combining the available data from all available log hazard functions, and independence of hazard functions and sampling designs. The second assumption is easily seen to be satisfied for our data. As for the other two assumptions, normality is not crucial and it has been shown in previous work that this method is robust to violations; furthermore, normality is expected to be approximately satisfied for the transformed mortalities in eq. (2) on which we base our analysis, and additional transformations towards normality can be easily implemented as needed. We note that the survival schedule itself is not assumed to be Gaussian. Since log hazard functions are assumed to change only

slowly, as calendar time progresses, it is unlikely that one encounters severe violations of the third assumption, although this assumption will not be strictly satisfied.

When the observations for each cohort are sparsely sampled or are incomplete, alternatives to the integral approximations (7) are needed, as these require densely sampled data over the entire domain (time, age). For cohorts with birth years within the last 80-100 years, their current and recent past mortality is not known, as mortality at older ages (e.g. for centenarians) has not been observed yet. A conditional expectation method has been developed in Yao et al. (2005) to address such situations of incompletely observed 
$\log$ hazard functions.

Let $\boldsymbol{Y}_{x_{P}^{*}}$ be the vector of observations (assembling the individual transformed central death rates as defined in eq. (2)) with the associated covariate value $x_{P}^{*}, \boldsymbol{Y}_{x_{P}^{*}}=$ $\left(Y_{x_{P}^{*}}\left(t_{1}\right), \ldots, Y_{x_{P}^{*}}\left(t_{n_{x_{P}^{*}}}\right)\right)^{\top}$, where $n_{x_{P}^{*}}$ is the number of available observations for this cohort. Let $\boldsymbol{\rho}_{k, x_{P}^{*}}$ be the vector of the values of the $k$ th eigenfunction, $\boldsymbol{\rho}_{k, x_{P}^{*}}=$ $\left(\rho_{k, x_{P}^{*}}\left(t_{1}\right), \ldots, \rho_{k, x_{P}^{*}}\left(t_{n_{x_{P}^{*}}}\right)\right)^{\top}, \boldsymbol{\Sigma}_{Y_{x_{P}^{*}}}$ be the covariance matrix of $\boldsymbol{Y}_{x_{P}^{*}}$, corresponding to the observed time points for the cohort, and $\boldsymbol{\mu}_{x_{P}^{*}}=E \boldsymbol{Y}_{x_{P}^{*}}$. For jointly Gaussian principal components $\xi_{k, x_{P}^{*}}$ and error terms $\varepsilon_{x_{P}^{*}, j}$, the conditional principal components are $E\left(\xi_{k, x_{P}^{*}} \mid \boldsymbol{Y}_{x_{P}^{*}}\right)=\lambda_{k, x_{P}^{*}} \boldsymbol{\rho}_{k, x_{P}^{*}}^{\top} \boldsymbol{\Sigma}_{Y_{x_{P}^{*}}}^{-1}\left(\boldsymbol{Y}_{x_{P}^{*}}-\boldsymbol{\mu}_{x_{P}^{*}}\right)$. The estimated conditional principal components are then obtained by substituting the corresponding estimates, leading to

$$
\hat{\xi}_{k, x_{P}^{*}}=\hat{\lambda}_{k, x_{P}^{*}} \hat{\boldsymbol{\rho}}_{k, x_{P}^{*}}^{\top} \hat{\boldsymbol{\Sigma}}_{Y_{x_{P}^{*}}}^{-1}\left(\boldsymbol{Y}_{x_{P}^{*}}-\hat{\boldsymbol{\mu}}_{x_{P}^{*}}\right) .
$$

These predicted principal components are then substituted into (11).

\subsection{Forecasting of Future Cohort Log Hazard Functions}

The proposed forecasting of mortality combines two principles: (a) Predicting incompletely observed log hazard functions and their principal components by conditional expectation, pooling information from across all log hazard functions as in (12); and (b) functional local linear extrapolation. For the latter, we adapt a proposal of Li and Heckman (2003) to obtain local linear extrapolation of the mean function, eigenfunctions, and principal components.

Cohorts close to year $x_{n}^{*}$ will contain incomplete information about the cohort hazard functions, due to the fact that members of such cohorts are still alive, so that their old-age mortality has not yet been observed. Keeping in mind the notational convention to denote target years for which prediction is needed by $x_{P}^{*}$ and target years for forecasting by $x_{F}^{*}$, we define prediction windows $\mathcal{W}\left(x_{P}^{*}\right)=\left[x_{P}^{*}-\tilde{\omega}, \min \left(x_{P}^{*}+\tilde{\omega}, x_{n}^{*}\right)\right]$, where $x_{P}^{*}$ is the covariate 
of the trajectory to be predicted. Here, $\tilde{\omega}$ needs to be chosen large enough so that (a) the set $\left\{t_{j}, 1 \leq i \leq n, 1 \leq j \leq n_{i} \mid x_{i} \in \mathcal{W}\left(x_{P}^{*}\right)\right\}$ is relatively dense in $[0, T]$; i.e., there are no "gaps", and (b) the set of all pairs $\left\{\left(t_{j}, t_{l}\right), 1 \leq i \leq n, 1 \leq j, l \leq n_{i} \mid x_{i} \in \mathcal{W}\left(x_{P}^{*}\right)\right\}$ is relatively dense in $[0, T] \times[0, T]$. These assumptions are typically satisfied for lifetime data if $\left(x_{P}^{*}-\tilde{\omega}\right)$ is small enough such that there is at least one $i$ with $x_{i} \geq x_{P}^{*}-\tilde{\omega}$ for which a complete cohort mortality trajectory on $[0, T]$ is available. For the Swedish cohort mortalities, we therefore chose $\tilde{\omega}=51$ years, such that for $x_{n}^{*}=1975$ we have $x_{n}^{*}-\tilde{\omega}=1924$, the last year for which completely observed cohort log hazard functions up to age 80 were available.

Choosing $L=L^{*}$ as determined in (A.1) and (A.2), the predicted log hazard functions over the entire domain $[0,80]$ for birth years $x_{P}^{*}$ are then given by

$$
\hat{Z}_{x_{P}^{*}}(t)=\hat{\mu}_{x_{P}^{*}}(t)+\sum_{k=1}^{L^{*}} \hat{\xi}_{k, x_{P}^{*}} \hat{\rho}_{k, x_{P}^{*}}(t) .
$$

Here $\left\{\hat{\xi}_{k, x_{P}^{*}}\right\}_{k=1, \ldots, L^{*}}$ are the predicted principal components, obtained through the conditional expectation approach (12), given incomplete cohort log hazard functions at $x_{P}^{*}$, and $\hat{\mu}_{k, x_{P}^{*}}$ and $\hat{\rho}_{k, x_{P}^{*}}$ are obtained for incompletely observed log hazard functions as described in Yao et al. (2005).

Next we consider forecasting log-hazard functions for cohorts born at a time $x_{F}^{*}, x_{F}^{*}>$ $x_{n}^{*}$, i.e., born after the last observed cohort. Given a forecasting window width $b>0$, the proposed forecast is

$$
\hat{Z}_{x_{F}^{*}}(t ; b)=\hat{\mu}_{x_{F}^{*}}(t ; b)+\sum_{k=1}^{L^{*}} \hat{\alpha}_{k}\left(x_{F}^{*} ; b\right) \hat{\rho}_{k, x_{F}^{*}}(t ; b),
$$

where extrapolation estimates $\hat{\mu}_{x_{F}^{*}}(t ; b), \hat{\rho}_{k, x_{F}^{*}}(t ; b)$ and $\hat{\alpha}_{k}\left(x_{F}^{*} ; b\right)$ are obtained as described in Appendix A.3. Discussion of the ideas underlying this forecasting approach can be found in Section 5.

Choice of a reasonable forecasting window width $b$ is important for the forecasting step. Related issues regarding bandwidth selection with correlated errors for exponential 
smoothing were discussed in detail by Gijbels, Pope and Wand (1999), where it was concluded that the historical averaged squared prediction error provides a good choice. We extend this idea of forward cross-validation in local linear extrapolation from the scatterplot setting ( $\mathrm{Li}$ and Heckman, 2003) to our forecasting problem. Details on this are provided in Appendix A.4, along with a description of the construction of bootstrap prediction intervals and the computational implementation of the procedures described in this paper.

\subsection{Predicting and Forecasting Swedish Cohort Log Hazard Functions}

Assume we wish to construct the mortality forecast for the Swedish 2000 cohort. In a preliminary prediction step we predict the log-hazard functions up to age 80 for the 1924-1975 cohorts which are only incompletely observed. For instance, we need to predict log-hazard functions at age 29 and later for the 1975 cohort, at age 30 and later for the 1974 year-of-birth cohort, and so on, using predictions (13). As an illustration, the predicted log-hazard function up to age 80 for the year 1975 birth cohort is displayed in Figure 5, superimposed on the observed raw $\log$-hazard functions $\log \left(\psi\left(\tilde{q}_{c}(t)\right)\right)$, which for this cohort are available up to age 29. The predicted 1975 trajectory is compared with the estimated trajectory for 1910 for which raw mortalities are available throughout. To obtain forecasts for log-hazard functions of more recent target years $x_{F}^{*}$, we couple these predictions with the proposed functional extrapolation (A.3) method described in Section 4.3 and Appendices A.3 and A.4.

The resulting forecasts for the functional components are illustrated in Figure 6 for the target year $x_{F}^{*}=2000$. The cross-validation forecasting window width $\hat{b}_{F C V}$ selected by (A.4) is seven. The grey curves for the mean $\hat{\mu}_{x}(t)$ and the eigenfunctions $\left\{\hat{\rho}_{k, x}(t)\right\}$, $x \in[1924,1975]$, at later ages (in lighter grey) are obtained by prediction. The resulting forecast for the log-hazard function of the 2000 cohort is demonstrated in Figure 7 , and 
compared with the estimated log-hazard functions for the 1800 and 1900 cohorts. We find that the forecast for the 2000 cohort implies an immediate sharp drop in the mortality rate at early ages, with the lowest mortality rate occurring around age 3 , followed by a steep increase in mortality to age 20 . Between ages 20 and 40, mortality rates stay more or less constant, and then steadily rise after age 40 .

Throughout, mortality rates of the 2000 cohort stay well below those of the 1800 and 1900 cohorts. This is supported by the $95 \%$ bootstrap prediction intervals that are included in the figure. Interestingly, the right tails of the log-hazard functions are roughly parallel and linear for all three cohorts, which suggests that the slope is invariant. Even more remarkable is that in the right tail the average distance of the log-hazard functions appears to be about the same between the three log-hazard functions. This suggests that the size of the decreases in old-age log-hazards has been exponentially increasing since 1800.

\section{DISCUSSION AND CONCLUDING REMARKS}

The moving windows approach has proved suitable for modeling mortality trends in population lifetables for Swedish women for the age range 0 to 80 . Birth year of the cohort serves as covariate, and other covariates as available could be included analogously. Our approach embodies the concept of time-varying principal components of mortality, and adequately models long-term mortality time-dynamics and changes in longitudinal cohort mortality as observed for the Swedish cohort lifetables. Extensions are proposed for the prediction of cohort log hazard functions for which complete log hazard functions have not been observed yet, and for the forecasting of future cohort mortality, when the target year is beyond the last available birth cohort data. These demographic projections are of central interest in demographic population studies, and of methodological interest in statistical demography. They are furthermore useful beyond demographic applications, whenever prediction and forecasting of partially observed log hazard functions, condition- 
ing on a continuous increasing covariate, is of interest.

We note that a key feature of the proposed forecasting of future cohort mortality is that it includes forecasting of both mean and random components. Accordingly, our forecast for a log-mortality function is based on forecasts of mean functions, eigenfunctions and principal components. Using extrapolation of the dependence structure in addition to the extrapolation of the mean is natural when one aims at forecasting the conditional expectation for a future log mortality function, given past observations on observed mortality. Within a window, as used in the log mortality fitting for completely observed cohort mortality, the log mortality functions are considered i.i.d. and the random components have zero expectation. Thus the departure of one log mortality curve from the mean does not contain information about nearby log mortality functions. However, this changes when moving forward into the future and away from the last windows containing cohorts with observed mortalities. Then the conditional expectation of the targeted future log mortality trajectories is not equal to the forecasts of the mean structure alone but does include random components. The evolution of the first eigenfunction, for example, provides information about the evolution of the conditional mean function, and it is prudent to include this information in the forecast. We thus base our forecast on predicting the overall stochastic structure (mean functions, eigenfunctions and principal components) of log-mortality from previous structure.

To validate our forecasting method, we also investigated forecasts for historical cohorts where the forecast is constructed assuming an analogous data situation as when forecasting the mortality for the 2000 cohort. A good diagnostic whether the forecast will work is provided by plots analogous to those provided in Figure 6, from which one can gauge whether a linear extrapolation of past observed trends makes sense. This is clearly the case for the year 2000, but is not the case for some years. Selecting as an example the year 1850, judging from the plot analogous to Figure 6 (not shown), the situation is less 
promising than for the year 2000 but the forecast still seems feasible. In the construction of the 1850 forecast, one pretends that the last available mortality rates are up to age 29 for the 1825 cohort and that the most recent cohort with completely observed mortality rates up to age 80 is the 1774 cohort. The comparison of the 1850 cohort log mortality forecast based on three components, including the $95 \%$ bootstrap confidence intervals, with the actual raw and fitted log-hazards based on the actual observed mortality data for the 1850 cohort, can be found in Figure 8. Overall, the forecast seems reasonably accurate, while it is also noteworthy that the reduction in older age mortality has accelerated and is underestimated in the forecast.

While the cohorts from which the lifetables are formed consist of different individuals, entire birth cohorts may be subject to period effects, which can appear as mortality shocks, caused, e.g., by epidemics, famines or war, changes in socioeconomic environments, or fluctuations in quality and availability of medical resources (for discussion of such period effects we refer to Fienberg and Mason, 1985, Fu, 2000, Yang, Fu and Land, 2004, and Yang et al., 2008). While each cohort will experience these effects at a different age range, they may have similar impact on cohort mortality for adjacent cohorts. We assume that in the model, these potential dependencies and period effects can be accounted for (a) by the similarity and continuity of cohort mortality trajectories in adjacent birth years, and (b) may be subsumed in measurement errors as included in model (2), and thus may be viewed as nuisance effects that can be neglected in the analysis. In practice, sporadic shocks will be averaged out during model fitting, while the longer-term slowly occurring changes are accounted for by the changing mean log hazard functions and eigenfunctions.

Advantages of our approach are, first, its inherent flexibility to adjust to arbitrarily shaped cohort log hazard functions, in contrast to popular parametric models which do not go beyond reflecting preconceived shapes and thus are not flexible enough for many demographic applications. Second, we propose extensions of this approach to the fore- 
casting of future cohort log hazard functions, also retaining a large degree of flexibility. Third, it provides novel insights into the covariance and correlation structure of mortality and the changes of this structure over time. Fourth, it leads to the useful concept of functional principal components of mortality which are also subject to change over time and provide an adequate decomposition of mortality. Fifth, it does not require complex iterative fitting algorithms and does not suffer from non-convergence as parametric models do, especially in cases where their fit is at best marginal.

In the analysis of Swedish mortality lifetable data the observed smooth time-variation of cohort log hazard functions, and of covariance and correlation surfaces of mortality, indicates that the basic assumptions of our approach are satisfied. We distinguish prediction of partially observed cohort log-hazard functions from forecasting of cohort log hazard functions for cohorts for which no data are available. Prediction is therefore a first step in the proposed forecasting method, which combines prediction with functional linear extrapolation.

We demonstrate prediction of log-hazard functions for the pre-1975 birth cohorts and forecasting for the more recent cohorts. Besides characteristic shape changes and sharp drops in the mortality of infants and younger adults that are revealed by the proposed approach, the forecast mortality is seen to uniformly decline at all ages, as birth year increases. A noteworthy feature is that the various log-hazard functions are linear and parallel at ages 60-80, suggesting exponentially increasing annual declines of log-hazards in these older age ranges. As with all extrapolation methods, caution needs to be exercised not to over-interpret the findings, as more fundamental changes in mortality may occur in the future, rendering the extrapolation algorithm less appropriate as it reaches further into the future.

In summary, functional data analysis methodology has much to offer for the study of mortality. It is useful for predicting and forecasting cohort log hazard functions, as well 
as for studying the internal dependence and covariance structure of log hazard functions for human populations.

\section{APPENDIX}

\section{A.1 Consistency of Estimated Cohort Log Hazard Functions}

We consider the case with no shrinkage where $\hat{\theta}=0$ in (10). We fix a bin center $x_{0}$ and assume the bin size is $\omega$, allowing for $\omega \rightarrow 0$ in the asymptotic setting where the spacing of the measurements is getting denser. One has $n_{\omega}$ i.i.d. lifetables for which $x_{i} \in\left[x_{0}-\omega, x_{0}+\omega\right]$, and for each of these lifetables, $n_{i}$ observations of mortality and corresponding log hazards as in (2).

Further assumptions include $n_{\omega} \rightarrow \infty, m \rightarrow \infty$ and, for the smoothing bandwidths $b_{\omega}$ used for mean and covariance function estimation (as described in the second paragraph in Section 2.2 and in more detail in Yao et al. $(2003,2005))$, that $b_{\omega}=o\left(n_{\omega}^{-1 / 4}\right), m b_{\omega} n^{-\delta_{1}} \rightarrow$ $\infty, m^{1-\delta_{2}} b_{\omega} \rightarrow \infty$ for $\delta_{1}, \delta_{2}>0$. Under conditions (C) in Section 3.1 of Hall, Müller and Wang (2006), slightly modifying the arguments in the proof of Theorem 3 of that paper and omitting indices $x_{0}$, one obtains that

$\left|\hat{\lambda}_{k}-\lambda_{k}\right|=O_{p}\left(n_{\omega}^{-1 / 2}\right), \quad \int\left(\hat{\rho}_{k}(t)-\rho_{k}(t)\right)^{2} d t=O_{p}\left(n_{\omega}^{-1}\right), \quad \int(\hat{\mu}(t)-\mu(t))^{2} d t=O_{p}\left(n_{\omega}^{-1}\right)$.

Considering the case where $L$ is known and fixed, using the representations (6) and (11), and omitting indices $x_{0}$, this leads to

$$
\begin{aligned}
& \int[\hat{Z}(t)-Z(t)]^{2} d t \\
& \leq C\left\{\int(\hat{\mu}(t)-\mu(t))^{2} d t+\sum_{k=1}^{L}\left\{\int\left(\hat{\rho}_{k}(t)-\rho_{k}(t)\right)^{2} d t+\left[\hat{\xi}_{k}-\xi_{k}\right]^{2} \int \rho_{k}(t)^{2} d t\right\}\right\}
\end{aligned}
$$

for a suitable constant $C>0$.

Working with the definitions (7) and (9), observing the smoothness assumptions and that $\tilde{\xi}_{k}=\hat{\xi}_{k}$ in the situation considered here, we find $\left(\hat{\xi}_{k}-\xi_{k}\right)^{2}=O_{p}\left(n_{\omega}^{-1}+m^{-1}\right)$ and 
obtain the asymptotic consistency result

$$
\int\left\{\hat{Z}_{x_{0}}(t)-Z_{x_{0}}(t)\right\}^{2} d t=O_{p}\left(n_{\omega}^{-1}+m^{-1}\right)
$$

This result demonstrates that integrated squared error of the fitted log-hazard functions converges to 0 asymptotically, with a rate of convergence that is determined by both the number of cohorts with birth year falling into the window and by the number of observations of mortality available per cohort.

\section{A.2 Choice of Bin Size and Number of Components}

We first determine a function $L^{*}(\omega)$ providing the optimal $L$ for each given bin size $\omega$. We define $L^{*}(\omega)$ to be the smallest value of $L$ such that when averaging over all bins, the first $L$ components explain $90 \%$ of the total variance. Formally,

$$
L^{*}(\omega)=\min \left\{L \geq 1: \quad \frac{1}{|\mathcal{J}|} \sum_{x_{0} \in \mathcal{J}} \frac{\sum_{k=1}^{L} \hat{\lambda}_{k x_{0}}}{\sum_{k=1}^{M} \hat{\lambda}_{k x_{0}}} \geq 0.90\right\}
$$

where $M$ is the largest number of components considered and the sum extends over all available bins with window width $\omega$, whose midpoints $x_{0}$ form the set $\mathcal{J}$, with $|\mathcal{J}|$ denoting the count of its elements. This method is computationally fast and the $90 \%$ level worked well in practice.

For each bin center $x_{0}$ and given bin size $\omega$, the empirical covariances are

$$
c_{i j l}\left(x_{0}, \omega\right)=\left(Y_{i}\left(t_{j}\right)-\hat{\mu}_{x_{0}}\left(t_{j}\right)\right)\left(Y_{i}\left(t_{l}\right)-\hat{\mu}_{x_{0}}\left(t_{l}\right)\right)
$$

for all $1 \leq j, l \leq m$ and $x_{i} \in \mathcal{W}\left(x_{0}\right)$, which are compared with the fitted covariances

$$
\widehat{c}_{j l}\left(x_{0}, \omega\right)=\sum_{k=1}^{L(\omega)} 1_{\left\{\hat{\lambda}_{k, x_{0}}>0\right\}} \hat{\lambda}_{k, x_{0}}(\omega) \hat{\rho}_{k, x_{0}}\left(t_{j}\right) \hat{\rho}_{k, x_{0}}\left(t_{l}\right)
$$

to determine the quality of the fit. The optimal bin size is then found by minimizing the sum of squared distances,

$$
\omega^{*}=\underset{\omega}{\arg \min }\left\{\sum_{x_{0}} \sum_{i: x_{i} \in \mathcal{W}\left(x_{0}, \omega\right)} \sum_{j, l=1}^{m}\left(c_{i j l}\left(x_{0}, \omega\right)-\widehat{c}_{j l}\left(x_{0}, \omega\right)\right)^{2}\right\} .
$$


Combining this with (A.1) yields the choice $L^{*}=L^{*}\left(\omega^{*}\right)$. In our application to Swedish mortality data, we include birth years $x_{0}=1760, \ldots, 1900$ in this optimization.

\section{A.3 Auxiliary Estimates for Mortality Forecasting}

These are computed in the following two steps:

Step 1 Functional principal component analysis based on moving windows: For a given bandwidth $b>0$, and each $x_{P}^{*} \in \mathcal{W}\left(x_{n}^{*}\right)=\left[x_{n}^{*}-b, x_{n}^{*}\right]$, applying (12) and the steps outlined before (13), obtain estimates $\left\{\hat{\mu}_{x_{P}^{*}}(t), \hat{\rho}_{k, x_{P}^{*}}(t), \hat{\xi}_{k, x_{P}^{*}}\right\}$, for all $k=1, \ldots, L$.

Step 2 Functional local linear extrapolation: Using the estimates from Step 1, obtain the following locally weighted least squares estimates for both functions as well as first derivatives, centering these estimates at $x_{n}^{*}$,

$$
\begin{gathered}
\left(\hat{\eta}_{k}^{(0)}, \hat{\eta}_{k}^{(1)}\right)=\underset{\left(\eta_{0}, \eta_{1}\right)}{\arg \min } \sum_{x_{P}^{*} \in \mathcal{W}\left(x_{n}^{*}\right)} K\left(\frac{x_{P}^{*}-x_{n}^{*}}{b}\right)\left\{\hat{\xi}_{k, x_{P}^{*}}-\eta_{0}-\eta_{1}\left(x_{P}^{*}-x_{n}^{*}\right)\right\}^{2}, \\
\left(\hat{\gamma}^{(0)}(t), \hat{\gamma}^{(1)}(t)\right) \underset{\left(\gamma_{0}, \gamma_{1}\right)}{\arg \min } \sum_{x_{P}^{*} \in \mathcal{W}\left(x_{n}^{*}\right)} K\left(\frac{x_{P}^{*}-x_{n}^{*}}{b}\right)\left\{\hat{\mu}_{x_{P}^{*}}(t)-\gamma_{0}-\gamma_{1}\left(x_{P}^{*}-x_{n}^{*}\right)\right\}^{2}, \\
\left(\hat{\beta}_{k}^{(0)}(t), \hat{\beta}_{k}^{(1)}(t)\right)=\underset{\left(\beta_{0}, \beta_{1}\right)}{\arg \min } \sum_{x_{P}^{*} \in \mathcal{W}\left(x_{n}^{*}\right)} K\left(\frac{x_{P}^{*}-x_{n}^{*}}{b}\right)\left\{\hat{\rho}_{k, x_{P}^{*}}(t)-\beta_{0}-\beta_{1}\left(x_{P}^{*}-x_{n}^{*}\right)\right\}^{2},
\end{gathered}
$$

for each $t=t_{j}$, where $t_{j} \in(0,1,2, \ldots, 80)$, and for $k=1, \ldots, L$. The kernel function $K(\cdot)$ is a symmetric density on domain $[-1,1]$, which we choose as $\frac{3}{4}\left(1-x^{2}\right)$ on $[-1,1]$. We obtain the predicted functions evaluated at time $x_{F}^{*}$ for each component by (functional) local linear extrapolations:

$$
\begin{aligned}
\hat{\alpha}_{k}\left(x_{F}^{*} ; b\right) & =\hat{\eta}_{k}^{(0)}+\left(x_{F}^{*}-x_{n}^{*}\right) \hat{\eta}_{k}^{(1)}, \\
\hat{\mu}_{x_{F}^{*}}(t ; b) & =\hat{\gamma}^{(0)}(t)+\left(x_{F}^{*}-x_{n}^{*}\right) \hat{\gamma}^{(1)}(t), \\
\hat{\rho}_{k, x_{F}^{*}}(t ; b) & =\hat{\beta}_{k}^{(0)}(t)+\left(x_{F}^{*}-x_{n}^{*}\right) \hat{\beta}_{k}^{(1)}(t),
\end{aligned}
$$

for all $t \in[0, T]$, where $T=80$ in our example. 


\section{A.4 Choice of Forecasting Window Width, Bootstrap Prediction Intervals and Computational Implementation}

Let $a=a\left(x_{n}^{*}, \delta\right)$, where $\delta=x_{F}^{*}-x_{n}^{*}$. The observed cohort log hazard functions with covariate values in $\left[a, x_{n}^{*}\right]$ are used as the cross-validation sample for selecting $b$. A reasonable choice is $a=x_{n}^{*}-r \delta$, where we adopt the suggestion of Li and Heckman (2003) to select $r=1$. Let $x_{j}$ be a birth year in $\left[a, x_{n}^{*}\right]$ for which we desire the cross-validated prediction error. The cross-validation forecast at $x_{j}$ is then solely based on data of cohorts born prior to $\left(x_{j}-\delta\right)$, leading to the forward cross-validation squared prediction error $\operatorname{FCV}(b)$

$$
\operatorname{FCV}(b)=\frac{1}{|\mathcal{I}|} \sum_{j \in \mathcal{I}} \int_{0}^{T}\left(\hat{Z}_{x_{j F}^{*}}(t ; b)-\hat{Z}_{x_{j}}(t)\right)^{2} d t .
$$

Here $\mathcal{I}=\left\{j: x_{j} \in\left[a, x_{n}^{*}\right]\right\},|\mathcal{I}|$ is the number of indices in $\mathcal{I}$ and $\hat{Z}_{x_{j F}^{*}}(t ; b)$ is obtained in the same way as in (14), except that $x_{F}^{*}=x_{j}$, i.e., the time frame is shifted back to $x_{j}$ or, equivalently, from $x_{n}^{*}$ to $\left(x_{j}-\delta\right)$, where $\hat{Z}_{x_{j}}$ may correspond to a fitted or predicted trajectory, depending on the value of $x_{j}$. In practice, $\mathrm{FCV}(b)$ is obtained via numerical approximation of the integral in (A.4). The cross-validation forecasting window width $\hat{b}_{F C V}$ is selected as minimizer of the squared prediction errors $\operatorname{FCV}(b)$,

$$
\hat{b}_{F C V}=\underset{b}{\arg \min } \operatorname{FCV}(b) .
$$

Prediction intervals were constructed with a bootstrap procedure based on resampling from suitable samples of cohort mortalities to form the bootstrap samples. The following summarizes this approach for forecast log-hazard functions:

$\left(B_{1}\right)$ Resample with replacement from the cohorts with $x_{i} \in\left[x_{n}^{*}-b, x_{n}^{*}\right]$ to form a bootstrap sample of the same size.

$\left(B_{2}\right)$ Use the bootstrap sample in $\left(B_{1}\right)$ to obtain the estimates $\hat{\mu}_{x_{F}^{*}}^{*}(t), \hat{\rho}_{k, x_{F}^{*}}^{*}(t ; b)$ and $\hat{\alpha}_{k}^{*}\left(x_{F}^{*} ; b\right)$ by the forecasting procedure in Section 4.3 , and obtain the bootstrap 
forecast at the future point $x_{F}^{*}$ in the same way as (14) but from the bootstrap sample.

$\left(B_{3}\right)$ Repeat steps $\left(B_{1}\right)-\left(B_{2}\right) B$ times to obtain $\hat{Y}_{x_{F}^{*}}^{*}(t)$. Construct the $95 \%$ pointwise prediction limits by finding the $2.5 \%$ and $97.5 \%$ quantiles of $\left\{\hat{Y}_{x_{F}^{*}}^{*}(t)\right\}$.

Regarding the computational implementation of the procedures, it is broken down into algorithmic modules (M1)-(M11) that are briefly described below. While the Matlab code can be found in the online Supplement, Matlab routines HADES and PACE for modules (M1), respectively (M2), which are basic for everything else, and are independently useful for many other applications, can be downloaded from http://anson.ucdavis.edu/ mueller/data/programs.html.

(A) Fitting and prediction of log-hazard functions: (M1) Compute nonparametric cohort-specific hazard rates through the transformation $\psi\left(\tilde{q}_{c}\right)$; (M2) Compute mean function, eigenfunctions and functional principal components, and also fitted covariance matrix, given a sample of functions (here applied to log hazard functions), and given the number of components $L$ to be included, both by the integration method (when the task is fitting) and by the conditioning method (12) (when the task is prediction); (M3) Construct windows $\mathcal{W}\left(x_{0}\right)$ and determine the included cohorts for each window, for all applicable $x_{0}$, given bin widths $\omega$; (M4) Call (M1)-(M3) repeatedly to find minimizers $L^{*}$, $\omega^{*}$ by iteratively solving (A.1) and (A.2); (M5) For fitting, use these minimizers for the final fits. These are obtained by calling (M2) for the selected cohorts for each $x_{0}$, applying the integration method, and obtaining as output the mean function (providing the fitted trajectory at $x_{0}$ ), eigenfunctions and principal components, and also the fitted covariance matrix; (M6) For prediction, assemble those cohorts falling into the (user-specified) prediction window $\mathcal{W}\left(x_{P}^{*}\right)$, then use $L^{*}$ from (M4) and call (M2) for all applicable $x_{P}^{*}$, applying the conditioning method. 
(B) Mortality forecasting: (M7) Given a forecasting bandwidth $b$, obtain fits/predictions, including the fitted/predicted mean functions, eigenfunctions and principal components, using the procedures in (A), for each $x_{P}^{*} \in \mathcal{W}\left(x_{n}^{*}\right)$ (see Step 1 Appendix A.3); (M8) Using the outputs from (M7), fit the locally weighted least squares estimates for each age and obtain the local linear extrapolations (A.3) for mean functions, eigenfunctions and principal components for each age $t$ and each cohort birth year $x_{F}^{*}$ for which forecasting is desired. Use these to compute the forecast for the log-hazard function; (M9) Assemble the fits/predictions obtained in (A) for all cohorts with birth years between $x_{n}^{*}-\left(x_{F}^{*}-x_{n}^{*}\right)$ and $x_{n}$, the forward cross-validation window, and then calculate mortality forecasts for these same cohorts, using for the forecasting only cohorts with birth years prior to $x_{n}^{*}-\left(x_{F}^{*}-x_{n}^{*}\right)$, by calling (M7) and (M8) with forecasting bandwidth $b$; (M10)

Find the optimal forecasting bandwidth $\hat{b}_{F C V}$ by minimizing the discrepancy between the forecasts and fits/predictions obtained in (M8), implementing (A.4) by numerical integration; (M11) Use $\hat{b}_{F C V}$ for the final mortality forecast, calling (M8).

\section{References}

[1] Ash, R. B., and Gardner, M. F. (1975), Topics in Stochastic Processes, New York: Academic Press, Inc.

[2] Booth, H. (2006), "Demographic Forecasting: 1980 to 2005 in Review," International Journal of Forecasting, 22, 547-581.

[3] Capra, W. B., and Müller, H. G. (1997), "An Accelerated-time Model for Response Curves," Journal of the American Statistical Association, 92, 72-83.

[4] Cardot, H. (2007), "Conditional Functional Principal Components Analysis," Scandinavian Journal of Statistics, 34, 317-335. 
[5] Chiou, J. M. and Müller, H. G. (2001) Discussion of "Inference of Density Families Using Functional Principal Component Analysis," Journal of the American Statistical Association, 96, 534-537.

[6] Chiou, J. M., Müller, H. G., and Wang, J. L. (2003), "Functional Quasi-likelihood Regression Models with Smooth Random Effects," Journal of the Royal Statistical Society, Ser. B, 65, 405-423.

[7] -- (2004), "Functional Response Models," Statistica Sinica, 14, 675-693.

[8] Congdon, P. (1993), "Statistical Graduation in Local Demographic Analysis and Projection," Journal of the Royal Statistical Society, Ser. A, 156, 237-270.

[9] Currie, I. D., Durban, M., and Eilers, P. HC (2004), "Smoothing and Forecasting Mortality Rates," Statistical Modelling, 4, 279-298.

[10] Debón, A., Montes, F., and Sala, R. (2006), "A Comparison of Models for Dynamic Life Tables. Application to Mortality Data from the Valencia Region," Lifetime Data Analysis, 12, 223-244.

[11] Dellaportas, P., Smith, A. F.M. and Stavropoulos, P. (2001), "Bayesian Analysis of Mortality Data," Journal of the Royal Statistical Society, Ser. A, 164, 275-291.

[12] Elandt-Johnson, R. C., and Johnson, N. L. (1980), Survival Models and Data Analysis, New York: John Wiley \& Sons.

[13] Faraway, J. J. (1997), "Regression Analysis for a Functional Response," Technometrics, 39, 254-262.

[14] Gijbels, I., Pope, A., and Wand, M. P. (1999), "Understanding Exponential Smoothing via Kernel Regression," Journal of the Royal Statistical Society, Ser. B, 61, 39-50.

[15] Glei, D., Lundström, H., and Wilmoth, J. (2006), "About Mortality Data for Sweden," Technical Report. Available at www.mortality.org or www.humanmortality.de. 
[16] Hall, P., Müller, H.-G., and Wang, J.-L. (2006), "Properties of Principal Component Methods for Functional and Longitudinal Data Analysis," The Annals of Statistics, 34, 1493-1517.

[17] Heligman, L., and Pollard, J. H. (1980), "The Age Pattern of Mortality," Journal of the Institute of Actuaries, 107, 49-80.

[18] Human Mortality Database. University of California, Berkeley (USA), and Max Planck Institute for Demographic Research (Germany). Available at www.mortality.org or www.humanmortality.de (data downloaded on 10 November, 2006).

[19] Fienberg, S. E. and Mason, W. M. (1985), "Specification and Implementation of Age, Period, and Cohort Models," in Cohort Analysis in Social Research, ed. W. M. Mason and S. E. Fienberg, New York: Springer-Verlag, pp. 45-88.

[20] Fu, W. J. (2000), "Ridge Estimator in Singular Design with Application to AgePeriod-Cohort Analysis of Disease Rates," Communications in Statistics, Part ATheory and Method, 29, 263-278.

[21] Hyndman, R. J., Koehler, A. B., Snyder, R. D., and Grose, S. (2002), "A State Space Framework for Automatic Forecasting Using Exponential Smoothing Methods," International Journal of Forecasting, 18, 439-454.

[22] Hyndman, R. J., and Ullah, M. S. (2007), "Robust Forecasting of Mortality and Fertility Rates: A Functional Data Approach," Computational Statistics and Data Analysis, 51, 4942-4956.

[23] Johnson, R.A., and Wichern, D.W. (2002),Applied Multivariate Statistical Analysis", New Jersey: Prentice Hall, Fifth Edition 
[24] Kneip, A., and Utikal, K.J. (2001), "Inference for Density Families Using Functional Principal Component Analysis," Journal of the American Statistical Association, 96, 519-532.

[25] Lee, R. D., and Carter, R. C. (1992), "Modeling and Forecasting U.S. Mortality," Journal of the American Statistical Association, 87, 659-671.

[26] Li, N., and Lee, R. (2005), "Coherent Mortality Forecasts for a Group of Populations: An Extension of the Lee-Carter Method," Demography, 42, 575-594.

[27] Li, N., Lee R., and Tuljapurkar S. (2004), "Using the Lee-Carter Method to Forecast Mortality for Populations with Limited Data," International Statistical Review, 72, $19-36$.

[28] Li, X., and Heckman, N. (2003), "Local Linear Extrapolation," Journal of Nonparametric Statistics, 15, 565-578.

[29] Müller, H. G. (2005), "Functional Modelling and Classification of Longitudinal Data (with Discussion)," Scandinavian Journal of Statistics, 32, 223-246.

[30] Müller, H. G., Chiou, J. M. and Leng, X. (2008) "Inferring Gene Expression Dynamics via Functional Regression," BMC Bioinformatics, 9, 60.

[31] Müller, H. G., Wang, J. L., and Capra, W. B. (1997), "From Lifetables to Hazard Rates: The Transformation Approach," Biometrika, 84, 881-892.

[32] Müller, H. G., and Zhang, Y. (2005), "Time-Varying Functional Regression for Predicting Remaining Lifetime Distributions from Longitudinal Trajectories," Biometrics, 61, 1064-1075.

[33] Oeppen, J., and Vaupel, J. W. (2002), "Broken Limits to Life Expectancy," Science, 296, 1029- 1031. 
[34] Park, Y., Choi J. W., and Kim, H.-Y. (2006), "Forecasting Cause-Age Specific Mortality Using Two Random Processes," Journal of the American Statistical Association, 101, 472-483.

[35] Ramsay, J., and Silverman, B. (2002), Applied Functional Data Analysis, New York: Springer.

[36] -- (2005), Functional Data Analysis (2nd ed.), New York: Springer.

[37] Renshaw, A., and Haberman, S. (2003), "Lee-Carter Mortality Forecasting: A Parallel Generalized Linear Modelling Approach for England and Wales Mortality Projections," Applied Statistics, 52, 119-137.

[38] Rice, J. (2004), "Functional and Longitudinal Data Analysis: Perspectives on Smoothing," Statistica Sinica, 14, 631-647.

[39] Rice, J., and Silverman, B. (1991), "Estimating The Mean and The Covariance Structure Nonparametrically When Data Are Curves," Journal of the Royal Statistical Society, Ser. B, 53, 233-243.

[40] Staniswalis, J. G., and Lee, J. J. (1998), "Nonparametric Regression Analysis of Longitudinal Data," Journal of the American Statistical Association, 93, 1403-1418.

[41] Vaupel, J. W., Carey, J. R., Christensen, K., Johnson T. E., Yashin, A. I., Holm, N. V., Iachine, I. A., Kannisto, V., Khazaeli, A. A., Liedo, P., Longo, V. D., Zeng,Y., Manton, K. G., and Curtsinger, J. W. (1998), "Biodemographic Trajectories of Longevity," Science, 280, 855-860.

[42] Vaupel, J. W., and Canudas Romo, V. (2003), "Decomposing Change in Life Expectancy: A Bouquet of Formulas in Honor of Nathan Keyfitzs 90th Birthday," Demography, 40, 201-216.

[43] Wang, J. L., Müller, H. G., and Capra, W. B. (1998), "Analysis of Oldest-Old Mortality: Lifetables Revisited," The Annals of Statistics, 26, 126-163. 
[44] Wong-Fupuy, C. and Haberman, S. (2004), "Projecting Mortality Trends: Recent Developments in the United Kingdom and the United States," Annals American Actuarial Journal , 8, 56-83.

[45] Yang, Y., Fu, W. J., and Land, K. C. (2004), "A Methodological Comparison of Age-Period-Cohort Models: Intrinsic Estimator and Conventional Generalized Linear Models," Sociological Methodology, 34, 75-110.

[46] Yang Y., Schulhofer-Wohl, S., Fu, W. J., and Land, K. C. (2008), "The Intrinsic Estimator for Age-Period-Cohort Analysis: What It Is and How To Use It, American Journal of Sociology, forthcoming.

[47] Yao, F., Müller, H. G., Clifford, A. J., Dueker, S. R., Follett, J. Lin, Y., Buchholz, B. A., and Vogel, J. S. (2003), "Shrinkage Estimation for Functional Principal Component Scores With Application to The Population Kinetics of Plasma Folate," Biometrics, 59, 676-685.

[48] Yao, F., Müller, H. G., and Wang, J. L. (2005), "Functional Data Analysis for Sparse Longitudinal Data," Journal of the American Statistical Association, 100, 577-590.

[49] Zhao, X., Marron, J. S., and Wells, M. T. (2004), "The Functional Data Analysis View of Longitudinal Data," Statistica Sinica, 14, 789-808. 
Table 1: Comparison of Leave-One-Curve-Out Prediction Errors Based On Global FPCA and Moving-Windows FPCA, for Various Numbers of Included Components.

\begin{tabular}{cccccccccc}
\hline \hline & \multicolumn{7}{c}{ Number of components $L$} & \\
Model & 0 & 1 & 2 & 3 & 4 & 5 & $\geq 6$ \\
\hline Full Window (Smooth) & & .0998 & .0282 & .0266 & .0256 & .0244 & .0239 & .0239 \\
Full Window & & .0998 & .0351 & .0349 & .0346 & .0347 & .0359 & .0358 \\
Moving Windows $^{c}$ & .0232 & .0230 & .0228 & .0227 & .0227 & .0227 & .0227 \\
\hline
\end{tabular}

${ }^{a}$ Functional quasi-likelihood regression model with smooth random effects using full window.

${ }^{b}$ Model (11) with the full window.

${ }^{c}$ Model (11) with the moving windows using the optimal bin size $\omega^{*}=11$. 


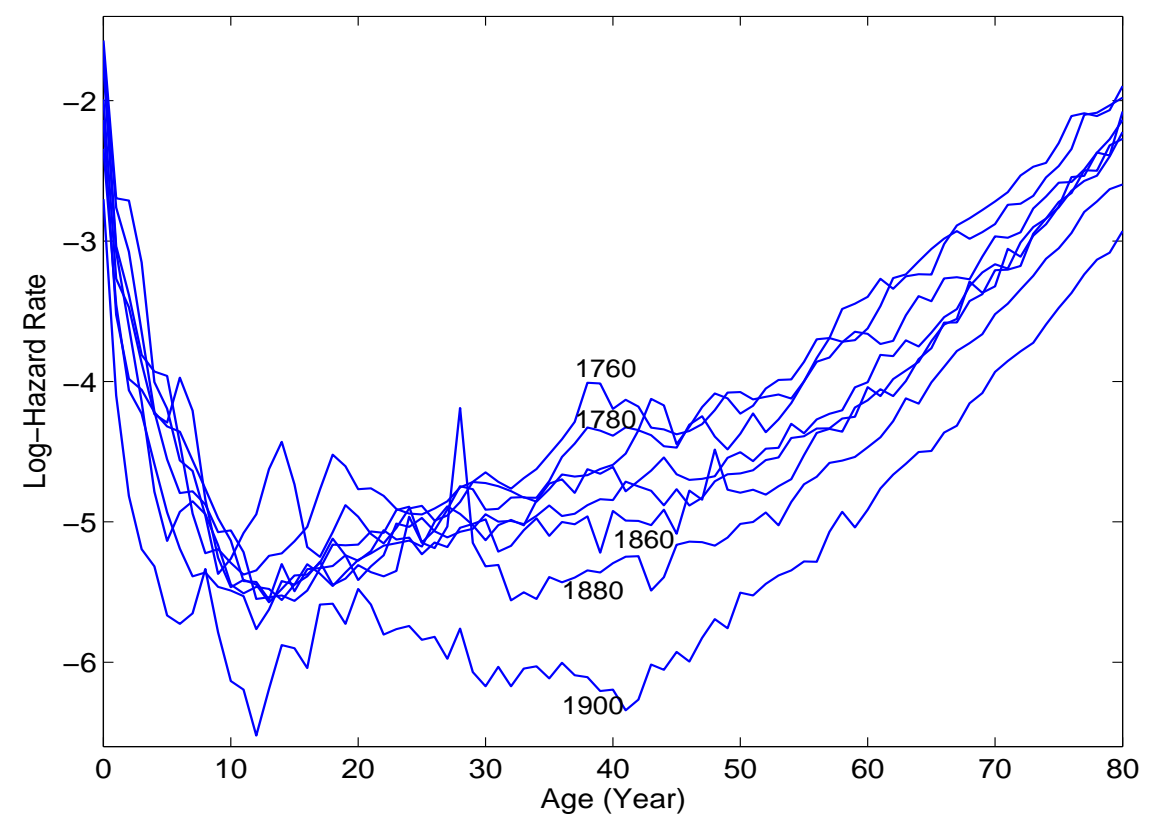

Figure 1: "Observed" log-hazard rates $Y(t)$ [eq. (2)] for cohorts born in 1760, 1780, 1800, 1820, 1840, 1860, 1880 and 1900, for Swedish lifetable data. 


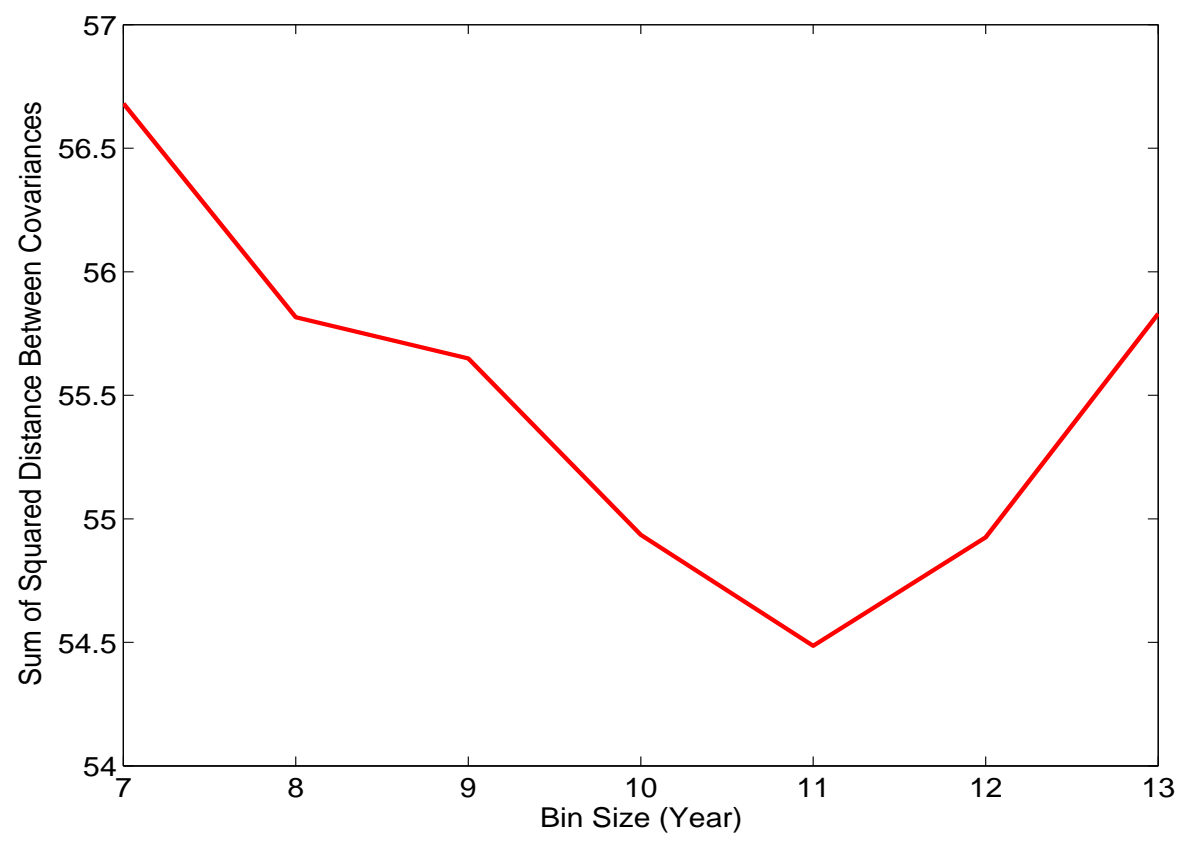

Figure 2: Choice of the optimal bin size $\omega^{*}$ [eq. (A.2)] for time-varying lifetable modeling: Sum of squared distance between the raw and the fitted covariances versus bin size for Swedish lifetable data. 

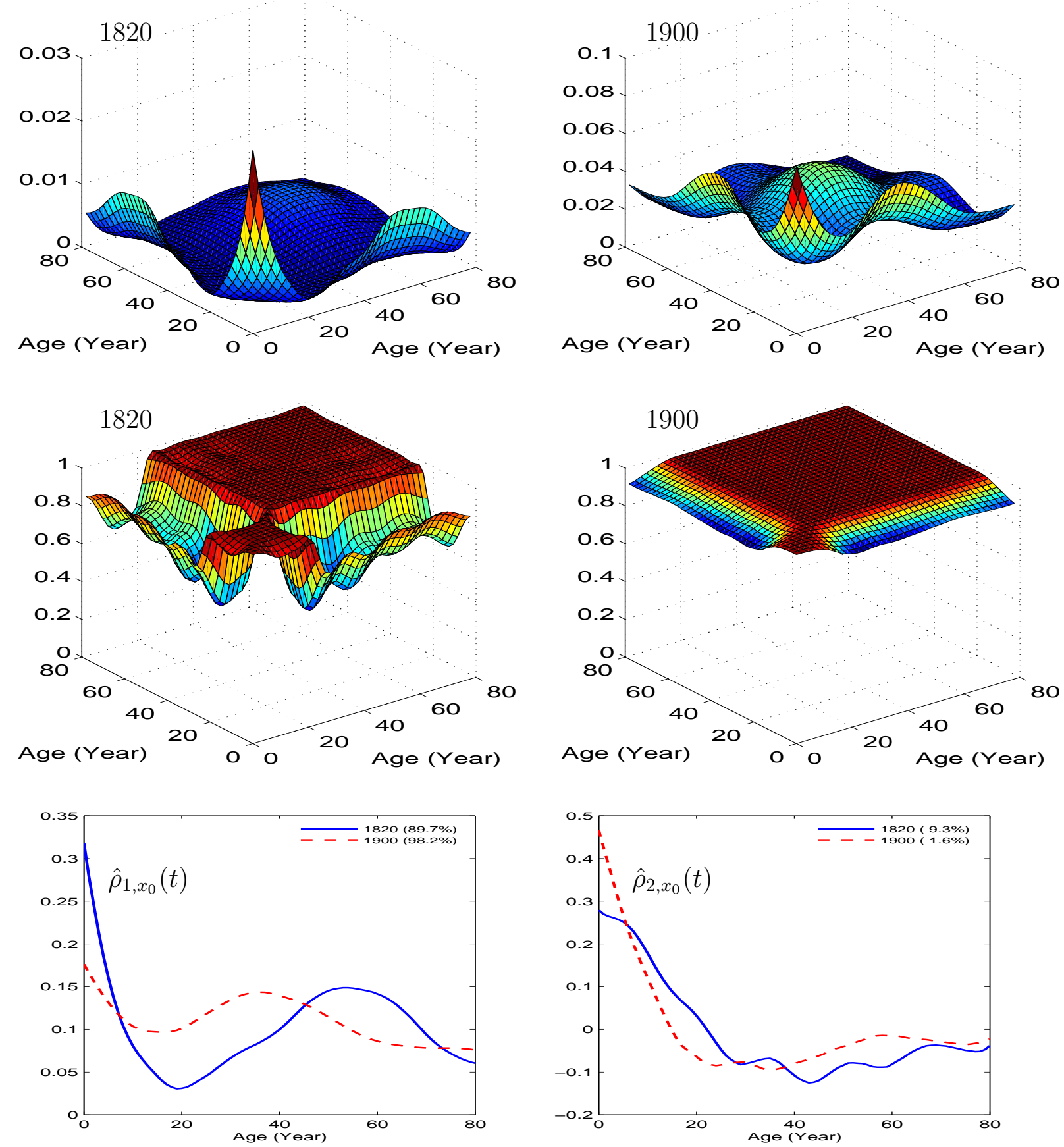

Figure 3: Upper and Middle panels: Estimated smooth covariance and fitted correlation surfaces, respectively, for cohorts born in $x_{0}=1820$ (left panel) and in $x_{0}=1900$ (right panel), using the moving windows approach with bin size $\omega^{*}=11$. Lower panels: First (left panel) and second (right panel) eigenfunctions, corresponding to the estimated covariance functions for 1820 and 1900. Percentages indicate fraction of variance explained by the respective component. 

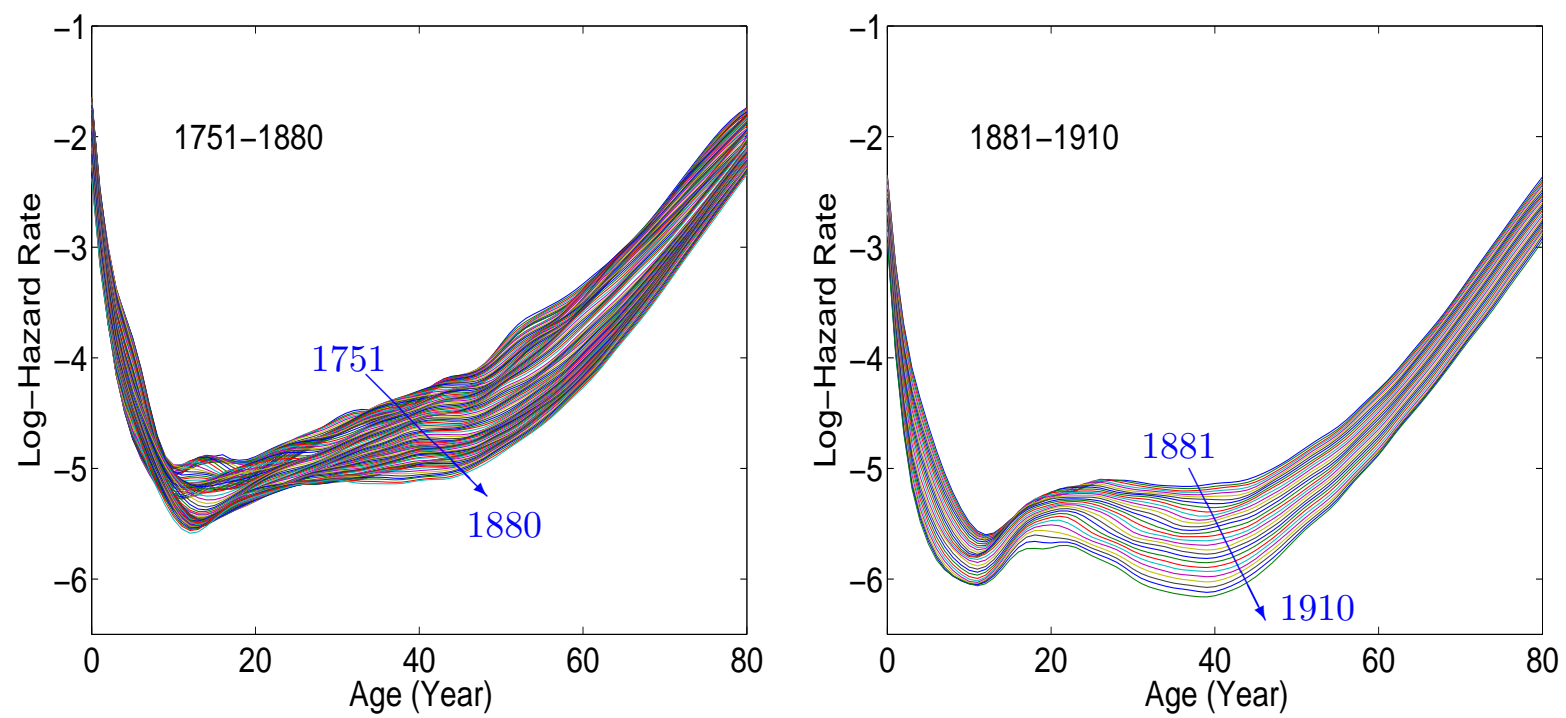

Figure 4: Fitted log-hazard functions $\hat{Z}_{x_{0}}\left[\right.$ eq. (11)] for cohorts born in $x_{0} \in[1751,1880]$ (left panel) and $x_{0} \in[1881,1910]$ (right panel). 


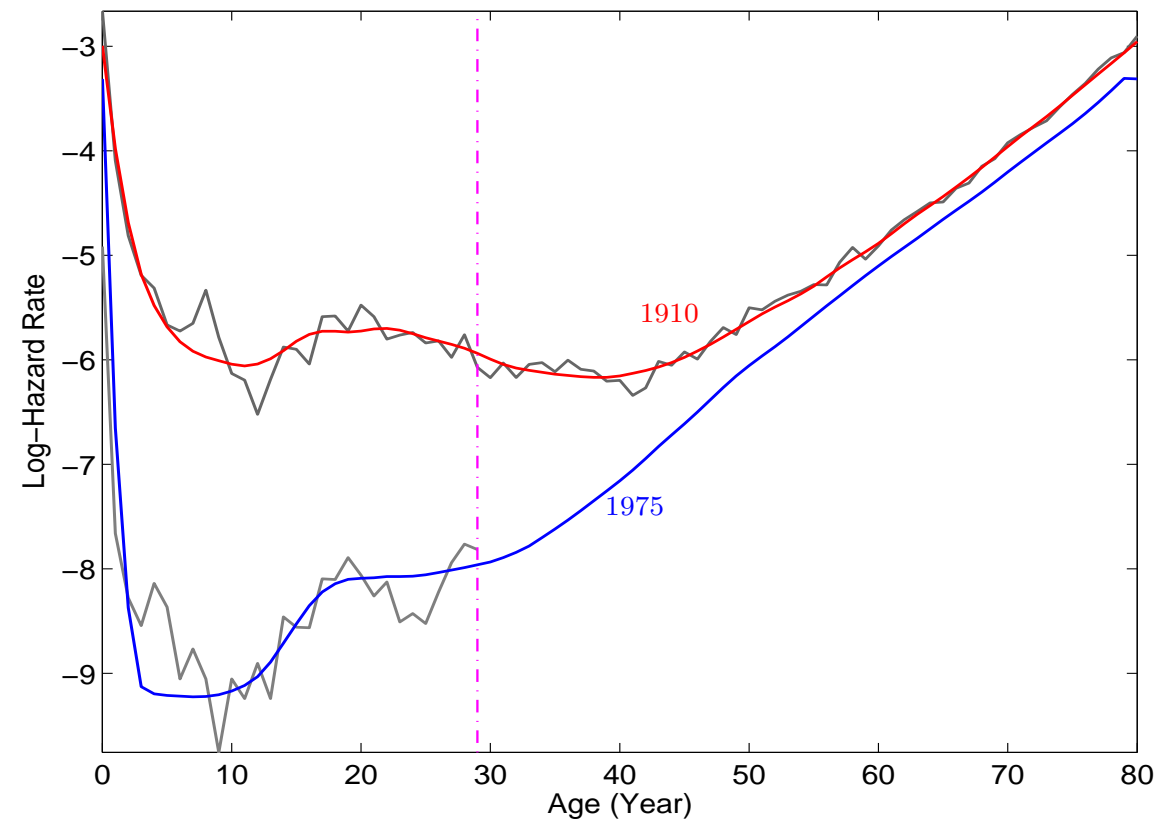

Figure 5: Predicted log-hazard function $\hat{Z}_{x_{P}^{*}}(t)$ [eq. (13)] for the 1975 cohort, where observed hazard rates $Y(t)$ are available up to age 29 only, in comparison with the fitted log-hazard function $\hat{Z}_{x_{0}}(t)$ [eq. (11)] for the 1910 cohort, for which observed hazard rates $Y(t)$ are available throughout lifetime. 

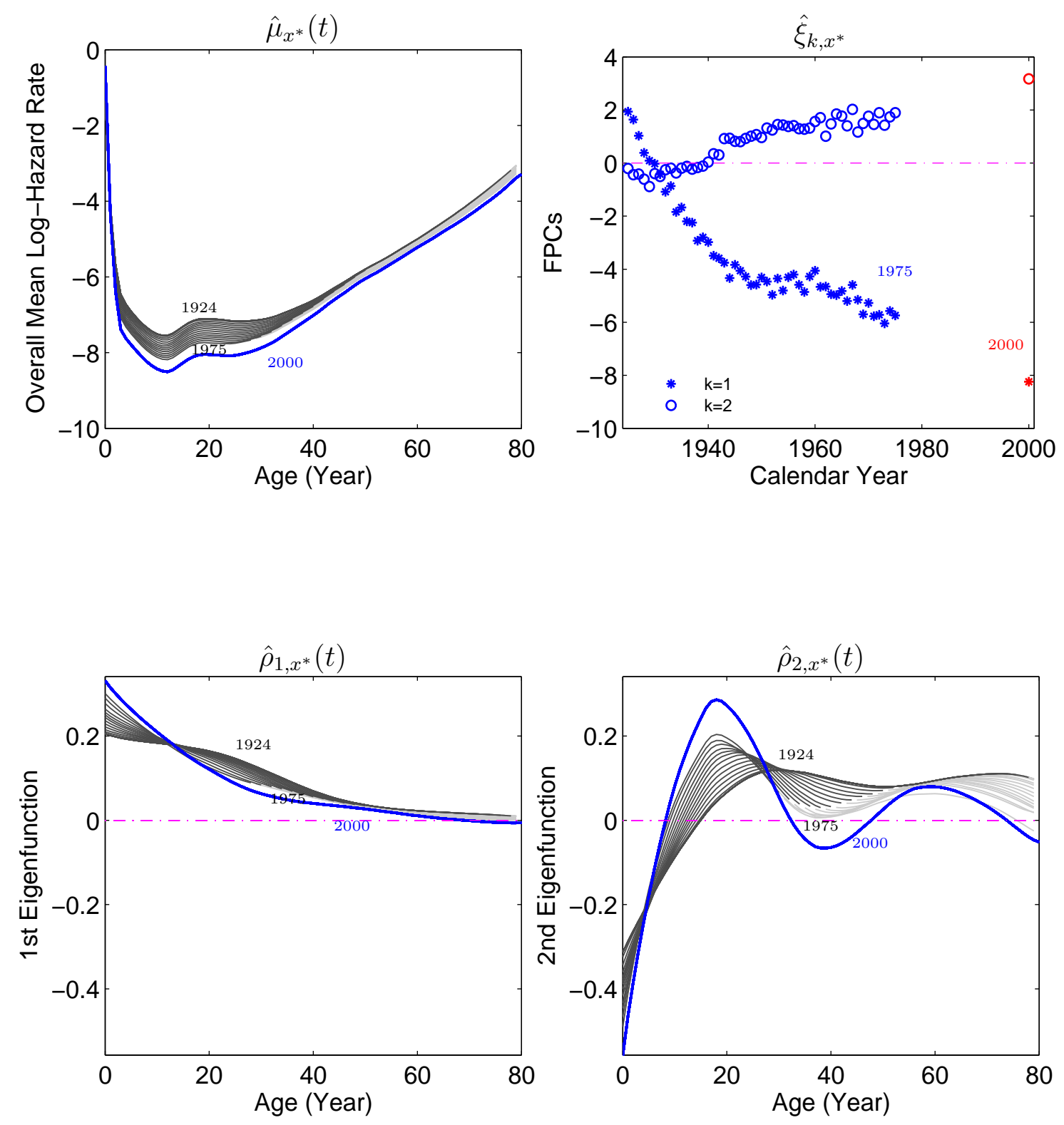

Figure 6: Predicted (for 1924-1975 cohorts) and forecast (for 2000 cohort) model components when using functional local linear extrapolation. Upper left panel: Predicted log hazard functions $\hat{\mu}_{x_{P}^{*}}(t)$ (gray) and forecast $\hat{\mu}_{x_{F}^{*}}(t)$ for the log hazard function of the 2000 cohort (black). Upper right panel: Predicted functional principal components $\left\{\hat{\xi}_{k, x_{P}^{*}}\right\}, x_{P}^{*} \in[1924,1975]$, for $k=1,2$ and forecast for the functional principal scores for the target year 2000 (rightmost points). Lower left panel: First eigenfunctions for the 1924-1975 cohorts (gray) and forecast $\hat{\rho}_{1, x_{F}^{*}}(t)$ of the first eigenfunction for the 2000 cohort (black). Lower right panel: Second eigenfunctions for the 1924-1975 cohorts (gray) and forecast $\hat{\rho}_{2, x_{F}^{*}}(t)$ of the second eigenfunction for the 2000 cohort (black). 


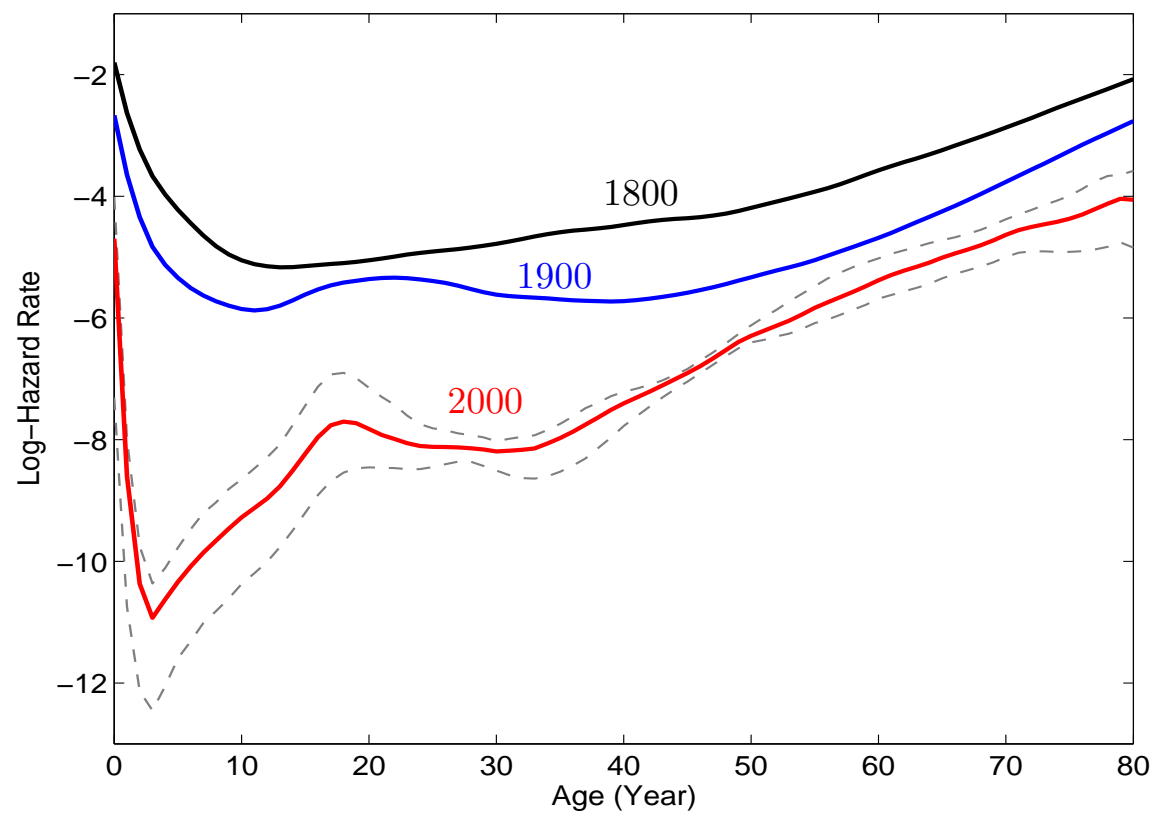

Figure 7: The forecast log-hazard function $\hat{Z}_{x_{F}^{*}}(t)$ [eq. (14)] with $95 \%$ bootstrap confidence intervals (dashed curve) for the 2000 cohort, using functional local linear extrapolation with $L^{*}=2$ random components, in comparison with the fitted log-hazard functions $\hat{Z}_{x_{0}}$ [eq. (11)] for the 1800 and 1900 cohorts. 


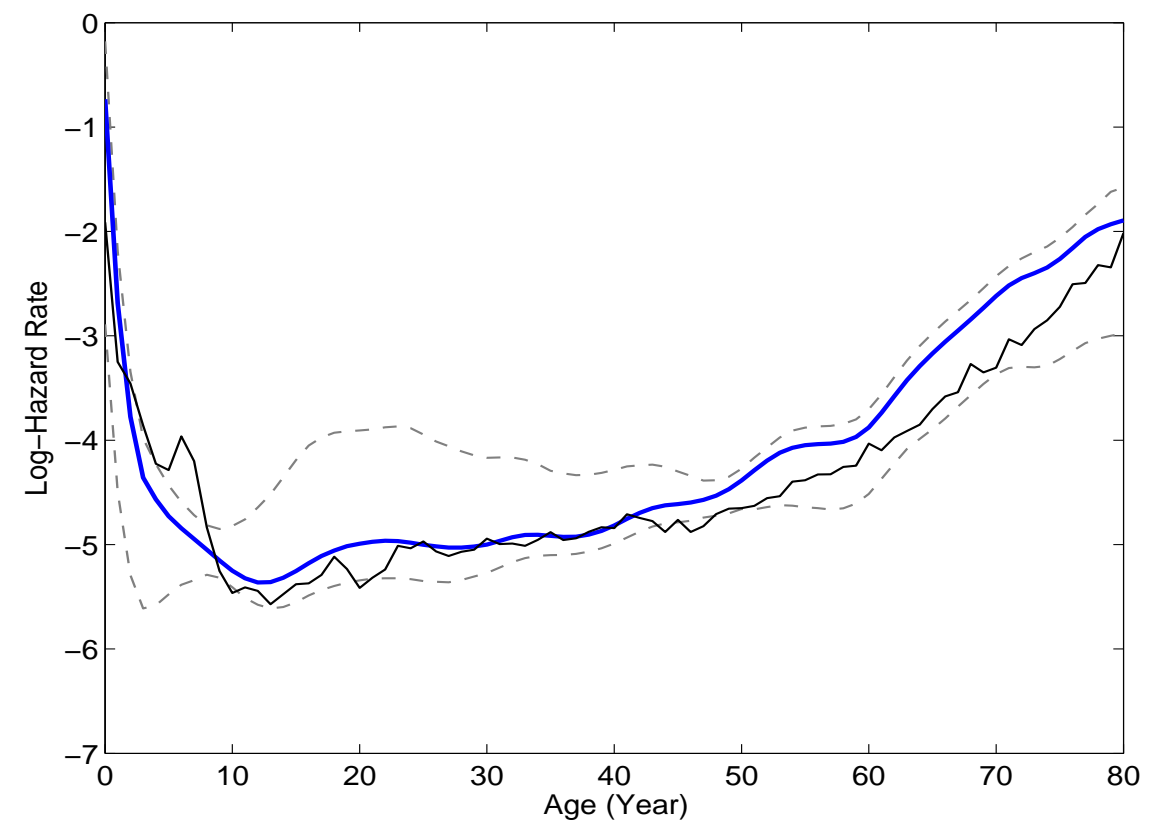

Figure 8: The forecast log-hazard function $\hat{Z}_{x_{F}^{*}}(t)$ [eq. (14)] for the 1850 cohort with $95 \%$ confidence intervals (dashed curve), pretending that the last available mortality rates are up to age 29 for the 1825 cohort and the most recent cohort with completely observed mortality rates up to age 80 is the 1774 cohort, in comparison with the observed log-hazard function for the 1850 cohort. 